\title{
Methanol maps of low-mass protostellar systems
}

\section{The Serpens molecular core}

\author{
L. E. Kristensen ${ }^{1}$, E. F. van Dishoeck ${ }^{1,2}$, T. A. van Kempen ${ }^{3}$, H. M. Cuppen ${ }^{1}$, C. Brinch ${ }^{1}$, \\ J. K. Jørgensen ${ }^{4}$, and M. R. Hogerheijde ${ }^{1}$ \\ 1 Leiden Observatory, Leiden University, PO Box 9513, 2300 RA Leiden, The Netherlands \\ 2 Max Planck Institut für Extraterrestrische Physik (MPE), Giessenbachstrasse 1, 85748 Garching, Germany \\ ${ }^{3}$ Harvard-Smithsonian Center for Astrophysics, 60 Garden Street, MS 78, Cambridge, MA 02138, USA \\ ${ }^{4}$ Centre for Star and Planet Formation, Natural History Museum of Denmark, Øster Voldgade 5-7, 1350 Copenhagen K., Denmark
} e-mail: kristensen@strw. leidenuniv.nl

Received 2 February 2010 / Accepted 2 April 2010

\section{ABSTRACT}

\begin{abstract}
Context. Methanol has a rich rotational spectrum providing a large number of transitions at sub-millimetre wavelengths from a range of energy levels in one single telescope setting, thus making it a good tracer of physical conditions in star-forming regions. Furthermore, it is formed exclusively on grain surfaces and is therefore a clean tracer of surface chemistry.

Aims. Determining the physical and chemical structure of low-mass, young stellar objects, in particular the abundance structure of $\mathrm{CH}_{3} \mathrm{OH}$, to investigate where and how $\mathrm{CH}_{3} \mathrm{OH}$ forms and how it is eventually released back to the gas phase.

Methods. Observations of the Serpens molecular core have been performed at the James Clerk Maxwell Telescope using the array receiver, Harp-B. Maps over a 4.5 $\times$ 5.4 region were made in a frequency window around $338 \mathrm{GHz}$, covering the $7_{K}-6_{K}$ transitions of methanol. Data are compared with physical models of each source based on existing sub-millimetre continuum data.

Results. Methanol emission is extended over each source, following the column density of $\mathrm{H}_{2}$ but showing up also particularly strongly around outflows. The rotational temperature is low, 15-20 K, and does not vary with position within each source. None of the Serpens Class 0 sources show the high- $K$ lines seen in several other Class 0 sources. The abundance is typically $10^{-9}-10^{-8}$ with respect to $\mathrm{H}_{2}$ in the outer envelope, whereas "jumps" by factors of up to $10^{2}-10^{3}$ inside the region where the dust temperature exceeds $100 \mathrm{~K}$ are not excluded. A factor of up to $\sim 10^{3}$ enhancement is seen in outflow gas, consistent with previous studies. In one object, SMM4, the ice abundance has been measured to be $\sim 3 \times 10^{-5}$ with respect to $\mathrm{H}_{2}$ in the outer envelope, i.e., a factor of $10^{3}$ larger than the gas-phase abundance. Comparison with $\mathrm{C}^{18} \mathrm{O} J=3-2$ emission shows that strong $\mathrm{CO}$ depletion leads to a high gas-phase abundance of $\mathrm{CH}_{3} \mathrm{OH}$ not just for the Serpens sources, but also for a larger sample of deeply embedded protostars.

Conclusions. The observations illustrate the large-scale, low-level desorption of $\mathrm{CH}_{3} \mathrm{OH}$ from dust grains, extending out to and beyond $7500 \mathrm{AU}$ from each source, a scenario which is consistent with non-thermal (photo-)desorption from the ice. The observations also illustrate the usefulness of $\mathrm{CH}_{3} \mathrm{OH}$ as a tracer of energetic input in the form of outflows, where methanol is sputtered from the grain surfaces. Finally, the observations provide further evidence of $\mathrm{CH}_{3} \mathrm{OH}$ formation through $\mathrm{CO}$ hydrogenation proceeding on grain surfaces in low-mass envelopes.
\end{abstract}

Key words. ISM: abundances - ISM: molecules - stars: formation - ISM: individual objects: Serpens

\section{Introduction}

A long-standing goal of astrochemistry has been to determine the physical and chemical conditions prevailing in star-forming regions (e.g., van Dishoeck \& Blake 1998). In this respect, different molecules act as tracers of different physical components, all depending on their formation history, their abundances, their chemical properties, etc. To effectively trace physical conditions such as density and temperature over the large range of values found in star-forming regions over the time-scale of starformation, it is of great importance to have as many independent tracers as possible. Methanol $\left(\mathrm{CH}_{3} \mathrm{OH}\right)$, with its rich rotational spectrum, is an excellent candidate tracing both temperature, density, grain surface formation and energy injection simultaneously during all phases of the early stages of stellar evolution.

$\mathrm{CH}_{3} \mathrm{OH}$ is a slightly asymmetric top molecule with numerous rotational transitions observable at millimetre- and sub-millimetre wavelengths. Because of the large number of transitions observable in a single frequency window it is possible to obtain a coherent data set very efficiently, making methanol a very suitable tracer of physical conditions, in particular in low-mass star forming regions where the emission is optically thin. Moreover, since the molecule is a slightly asymmetric top molecule, it traces very efficiently both density and temperature (e.g., Maret et al. 2005; Jørgensen et al. 2005b; Leurini et al. 2007).

Methanol forms exclusively on ice-covered dust grain surfaces primarily through hydrogenation of $\mathrm{CO}$ (Watanabe \& Kouchi 2002; Fuchs et al. 2009). Observations of interstellar ices show that methanol is indeed a prominent ice component, with abundances of up to almost $30 \%$ with respect to solidstate $\mathrm{H}_{2} \mathrm{O}$ or a few $\times 10^{-5}$ with respect to gas-phase $\mathrm{H}_{2}$ (e.g., Dartois et al. 1999; Gibb et al. 2004; Pontoppidan et al. 2004). In contrast, pure gas phase reactions produce negligible $\mathrm{CH}_{3} \mathrm{OH}$ abundances of less than $10^{-10}$ (Garrod et al. 2006). The question that naturally arises is how methanol desorbs from the surface 
of a dust grain to be observed in the gas phase. Is it through thermal heating of the entire grain, or is it through non-thermal desorption, where cosmic rays, UV-photons or exothermic reactions provide local heating of the grain? The former mechanism is at play close to young stellar objects (YSOs), in the inner-most part of the molecular envelope where the gas temperature exceeds $100 \mathrm{~K}$ (van Dishoeck et al. 1995; Ceccarelli et al. 2000; van der Tak et al. 2000; Schöier et al. 2002; Maret et al. 2005; Jørgensen et al. 2005b) and in outflows where hot gas sputters the icy mantles (e.g. Bachiller et al. 1995; Bachiller \& Pérez Gutiérrez 1997). This mechanism allows for an effective methanol enrichment of the environment and abundances are typically in the range of $10^{-7}$ to $10^{-6}$. The non-thermal mechanism dominates in cold, dark clouds and the outer parts of molecular envelopes (Hasegawa \& Herbst 1993; Herbst \& Cuppen 2006; Garrod et al. 2007; Öberg et al. 2009a). Here, reported abundances typically have values of $10^{-10}$ to $10^{-8}$. Hence, methanol also acts as a tracer of energetic processes in starforming regions.

So far most studies have concentrated on spectra at a single position or at most a few around them. Recently, large-scale mapping of weak molecular lines has become very efficient with the advent of array receivers such as the 16 pixel Harp-B receiver on the James Clerk Maxwell Telescope (JCMT). This allows for a direct study of the entire protostellar system (primarily envelope and outflow) on scales of several arcminutes at $15^{\prime \prime}$ resolution and for determination of density, temperature and energy input into the system. These observations will eventually be compared directly to observations of another important grain surface product, $\mathrm{H}_{2} \mathrm{O}$, to be done with the Herschel Space Observatory. By mapping the entire region, comparison with the different Herschel-beams $\left(9^{\prime \prime}-40^{\prime \prime}\right)$ will be straight-forward.

The Serpens molecular core (also known as cluster A) is located at a distance of $230 \pm 20 \mathrm{pc}$, following the discussion in Eiroa et al. (2008). The Serpens molecular core consists of several deeply embedded sources, of which at least four are identified as containing protostars (Wolf-Chase et al. 1998; Hogerheijde et al. 1999), SMM1, SMM3, SMM4 and S68N. Large-scale continuum-emission studies have been performed to quantify the spectral energy distribution of all sources in order to classify their evolutionary stage as well as the dust properties of the molecular envelopes surrounding each source (e.g., Casali et al. 1993; Hurt \& Barsony 1996; Testi \& Sargent 1998; Davis et al. 1999; Larsson et al. 2000; Williams \& Myers 2000), most recently with the Spitzer Space Telescope as part of the Cores to Disks legacy program (c2d; Harvey et al. 2007; Evans et al. 2009). These studies show that three of the sources (SMM3, SMM4 and S68N) have relatively low bolometric luminosities of $\sim 5 L_{\odot}$ each, whereas SMM1 has a higher luminosity of $\sim 30 L_{\odot}$ (e.g., Hogerheijde et al. 1999; Larsson et al. 2000). The mass of each system (envelope and star) is in all cases less than $10 M_{\odot}$. Recent interferometer observations by Choi (2009) show that SMM1 is a binary system with a projected separation of $\sim 500$ AU. The binary SMM1b appears less embedded than the primary (SMM1a). This discovery has been refuted by Enoch et al. (2009) and van Kempen et al. (2009) who both resolve the disk around SMM1. Through detailed SED modelling, Enoch et al. (2009) finds a very high disk mass of $\sim 1 M_{\odot}$ and that the inner parts of the envelope have been cleared out to distances of $500 \mathrm{AU}$.

The region has been studied extensively at millimetre (mm) and sub-millimetre (sub-mm) wavelengths in numerous molecular transitions (e.g., McMullin et al. 1994; White et al. 1995; Wolf-Chase et al. 1998; Hogerheijde et al. 1999;
McMullin et al. 2000), however it was not included in the molecular surveys of isolated Class 0 and I objects in Perseus and Ophiucus (Jørgensen et al. 2004, 2005b; Maret et al. 2005). The previous molecular studies conclude that SMM1, SMM3, SMM4 and S68N are all very similar to other young, low-mass stars with similar luminosities, such as IRAS 16293-2422 and NGC 1333 IRAS4A and 4B in terms of abundances of simple molecules that may be formed directly in the gas phase, e.g., $\mathrm{HCO}^{+}, \mathrm{CS}, \mathrm{HCN}$ (McMullin et al. 1994; Hogerheijde et al. 1999; McMullin et al. 2000). In Serpens, little has been done to quantify excitation and gas-phase abundances of molecules predominantly formed on dust grain surfaces, like $\mathrm{CH}_{3} \mathrm{OH}$, even though several lines have been detected by McMullin et al. (1994, 2000) and Hogerheijde et al. (1999).

More direct observations of grain surface products have been made by Pontoppidan et al. (2004), who mapped infrared absorption by molecules in the ice over a region extending $40^{\prime \prime}$ south of SMM4, but still located well within the molecular envelope. The primary ice constituents were found to be $\mathrm{H}_{2} \mathrm{O}, \mathrm{CO}$ (0.4-0.9 with respect to $\left.\mathrm{H}_{2} \mathrm{O}\right), \mathrm{CH}_{3} \mathrm{OH}(0.28$ with respect to $\left.\mathrm{H}_{2} \mathrm{O}\right)$ and $\mathrm{CO}_{2}\left(0.3-0.5\right.$ with respect to $\mathrm{H}_{2} \mathrm{O}$; Pontoppidan et al. 2008). The solid-state $\mathrm{CH}_{3} \mathrm{OH}$ abundance is one of the highest reported to date, both when compared to $\mathrm{H}_{2} \mathrm{O}$ but also with respect to gas-phase $\mathrm{H}_{2}\left(3 \times 10^{-5}\right)$. At distances greater than $12000 \mathrm{AU}$ the $\mathrm{CH}_{3} \mathrm{OH}$-ice abundance drops beneath the detection limit, corresponding to $3 \times 10^{-6}$ with respect to $\mathrm{H}_{2}$.

Besides the protostellar objects themselves, the region is permeated by large-scale outflows extending several arcminutes from the different sources with $\mathrm{CO} J=2-1$ velocities ranging from $\pm 10-15 \mathrm{~km} \mathrm{~s}^{-1}$ with respect to $v_{\mathrm{LSR}}=8-8.5 \mathrm{~km} \mathrm{~s}^{-1}$ (Davis et al. 1999; Graves et al. 2010). Garay et al. (2002) observed the outflows from SMM4 and $\mathrm{S} 68 \mathrm{~N}$ in $\mathrm{CH}_{3} \mathrm{OH} 3_{K}-2_{K}$ emission and inferred $\mathrm{CH}_{3} \mathrm{OH}$ column densities of $1-2 \times 10^{15} \mathrm{~cm}^{-2}$, corresponding to molecular abundance enhancements of $\sim 50-330$, depending on outflow position, consistent with studies of other outflows (e.g., Bachiller et al. 1995, 1998).

Here, the first map of rotationally excited methanol in the Serpens Molecular Core is presented of transitions which cover the energy range of $E_{\text {up }} \sim 65-115 \mathrm{~K}$. The paper is structured as follows. In Sect. 2 the observations are presented, and in Sect. 3 the observational results are provided along with radiative transfer modelling. Section 4 presents a discussion of the results, with a particular focus on the formation, desorption and excitation processes. Section 5 concludes the paper.

\section{Observations}

Observations of the Serpens molecular core were performed on June 20-22 2008 with the James Clerk Maxwell Telescope ${ }^{1}$ (JCMT) on Mauna Kea, Hawaii. Observations were made of the $7_{K}-6_{K}$ rotational band of methanol $\left(E_{\mathrm{up}} \sim 65-115 \mathrm{~K}\right)$ at frequencies ranging from 338 to $339 \mathrm{GHz}$ using the Harp-B array receiver consisting of $4 \times 4$ individual receivers (Smith et al. 2003). The telescope was pointed at two different locations near Serpens SMM1 and two different locations near SMM4, see Fig. 1, covering a total extent of $4.5 \times 5.4$. Observations were also made of $\mathrm{C}^{18} \mathrm{O}, J=3-2$ at $329.330 \mathrm{GHz}$ in a similar fashion, so as to be able to compare methanol and $\mathrm{CO}$ emission. Observations were made in jiggle-mode to achieve full spatial

\footnotetext{
1 The James Clerk Maxwell Telescope is operated by the Joint Astronomy Centre on behalf of the Science and Technology Facilities Council of the United Kingdom, the Netherlands Organisation for Scientific Research and the National Research Council of Canada.
} 
L. E. Kristensen et al.: Methanol maps of low-mass protostellar systems. I.

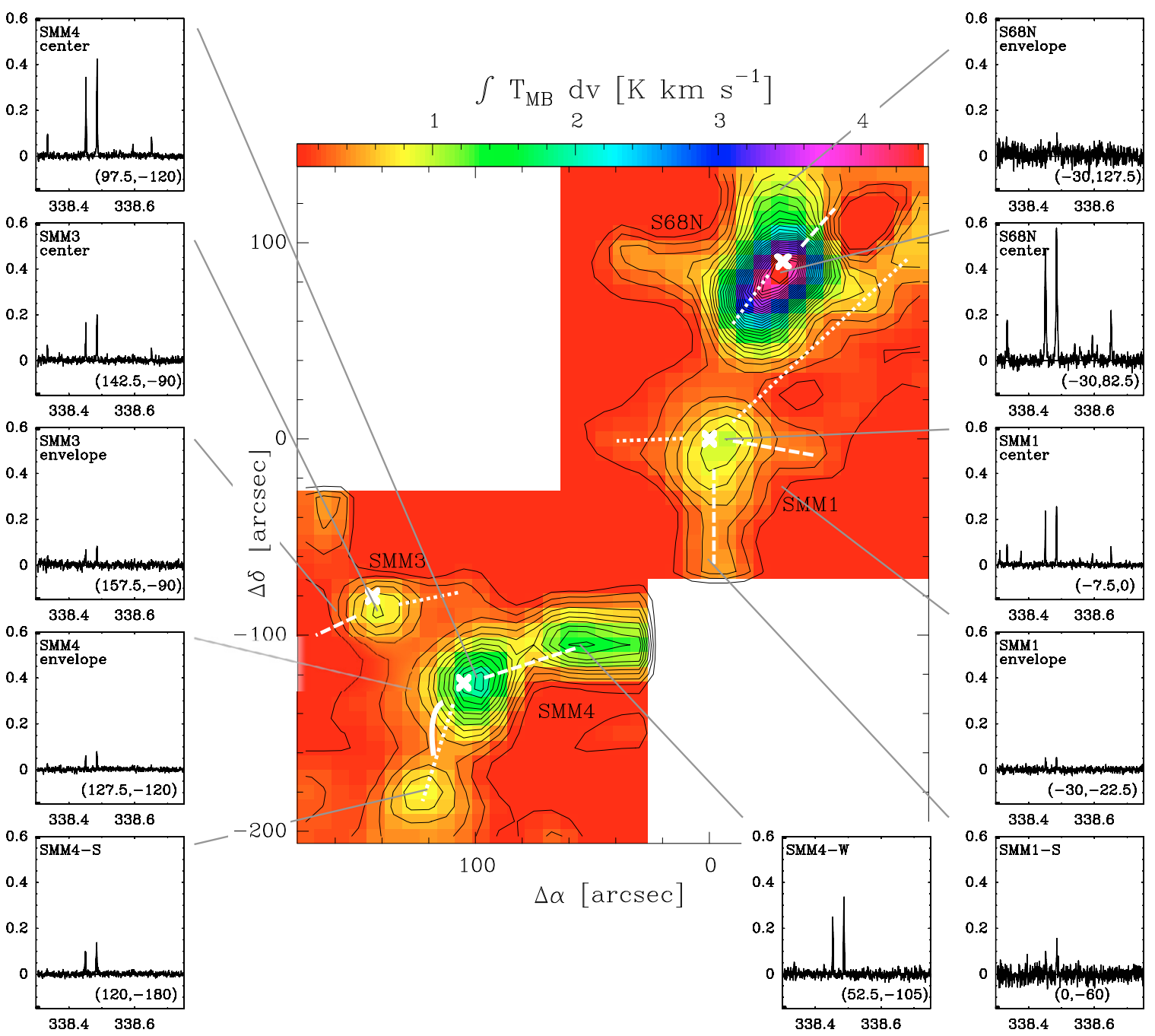

Fig. 1. Integrated emission, $\int T_{\mathrm{MB}} \mathrm{d} v$, of the strong $7_{0}-6_{0} \mathrm{~A}^{+}$line at $338.41 \mathrm{GHz}$. Objects Serpens SMM1, 3, 4 and S68N are labelled. Offsets are in arcsec with respect to SMM1. Contours are at $3 \sigma, 6 \sigma, 9 \sigma$, etc. The units for colour are $\mathrm{K} \mathrm{km} \mathrm{s}^{-1}$. The pixel size is $7.5 \times 77^{\prime \prime} 5$. The lines indicate outflow directions, with dashed representing blue-shifted outflow emission, and dotted lines representing red-shifted emission. The full white line extending south of SMM4 depicts the location of the four stars used by Pontoppidan et al. (2004) to measure the $\mathrm{CH}_{3} \mathrm{OH}$-ice abundance. Shown are also sample spectra at different positions, illustrated by triangles, from a single spatial pixel and provided in each inset in units of arcsec. The abscissa is for frequency in units of $\mathrm{GHz}$ and the ordinate is for main beam temperature in units of $\mathrm{K}$. All spectra have the same scale to ease intercomparison.

Nyquist-sampling using the Harp4 jiggle-pattern over a $2^{\prime} \times 2^{\prime}$ region. The beam-size of the JCMT at $338.5 \mathrm{GHz}$ is $\sim 15^{\prime \prime}$ and data were subsequently put on a map with a pixel-size of 7 ". 5 , i.e., one half beam-size to fulfill the Nyquist sampling criterion. The methanol observations were done using beam-switch with a throw of $180^{\prime \prime}$ in azimuth while the $\mathrm{C}^{18} \mathrm{O}$ observations were done using position switch to a clean position $1^{\circ}$ off. The weather at the time of the observations was good with $\tau(225 \mathrm{GHz}) \leq 0.1$. Pointing and calibration were checked at regular intervals, and the calibration error is estimated to be $\$ 20 \%$ based on a comparison with standard calibration sources. Data were brought from the antenna temperature scale, $T_{A}^{*}$, to the main beam temperature scale, $T_{\mathrm{MB}}$, by dividing with the main beam efficiency, $\eta_{\mathrm{MB}}$, which is 0.60 for the JCMT at these frequencies. The spectral resolution of the $\mathrm{CH}_{3} \mathrm{OH}$ spectra is $0.43 \mathrm{~km} \mathrm{~s}^{-1}$, and the $\mathrm{C}^{18} \mathrm{O}$ spectra were rebinned to the same value.

The mean rms in the methanol spectra is $20 \mathrm{mK}$ in $0.43 \mathrm{~km} \mathrm{~s}^{-1}$ velocity bins over the entire map. The $1 \sigma$ noise level on integrated emission has been determined as $1.2 \sigma_{\text {rms }} \sqrt{F W Z I \times \delta v}$ where the factor 1.2 accounts for the $20 \%$ telescope calibration uncertainty, $\sigma_{\text {rms }}$ is the rms noise, $F W Z I$ the estimated full width at zero intensity (taken to be $5 \mathrm{~km} \mathrm{~s}^{-1}$ ) and $\delta v$ the velocity bin $\left(0.43 \mathrm{~km} \mathrm{~s}^{-1}\right)$. The $3 \sigma$ uncertainty is typically $\sim 0.1 \mathrm{~K} \mathrm{~km} \mathrm{~s}^{-1}$ per spatial pixel, but increases near the edges of the map by a factor of $2-3$.

Initial data reduction was done using the Starlink package. This consisted of putting the spectra on to a regular grid with pixel-size 7.'5, subtracting linear baselines and co-adding all data-cubes. The Gildas package CLASS was used for subsequent analysis. Standard data reduction revealed emission in the off-position for a subset of the methanol observations, but it was possible to remove this emission due to two facts: (1) the off-emission never coincided with on-emission, i.e., no doublepeaked or "self-absorbed" line profiles were observed. In general the velocity offset between emission and absorption lines was of the order of $\sim 5 \mathrm{~km} \mathrm{~s}^{-1}$ compared to typical line-widths of 3-4 $\mathrm{km} \mathrm{s}^{-1}$ in the region where the off-emission was seen. (2) Data were recorded with a shared off-position, that is, even though the telescope jiggled on the on-position, no jiggling was done on the off-position. Therefore it was possible to recreate 
the off-emission in the strongest emission line at $338.409 \mathrm{GHz}$ in the following way: first the strongest "absorption" line was fitted with a Gaussian profile, then an artificial spectrum was created assuming a rotational temperature of $15 \mathrm{~K}$, which was added to each spectrum. The spectra were then analysed and no absorption features remained within the noise limit.

\section{Results}

In all spectra emission lines from the $7{ }_{K}-6_{K}$ rotational band of methanol are detected both on and off sources. In the central parts of each of the four YSOs up to eight emission lines are detected originating from ten transitions. The strongest line, the $7{ }_{0}-6_{0} \mathrm{~A}^{+}$line at $338.409 \mathrm{GHz}$, has a peak brightness temperature of up to $0.5 \mathrm{~K}$. All line profiles are Gaussian, no line asymmetries due to outflow activity or infall are observed. The $F W H M$ of the emission lines are $\sim 4-6.5 \mathrm{~km} \mathrm{~s}^{-1}$, consistent with other observations of methanol line widths (e.g. Maret et al. 2005; Jørgensen et al. 2005b). Line widths change between objects, but remain constant within each object, i.e., line widths do not change with position within an envelope. In Fig. 1 an overview is provided of the integrated emission in the strong $7_{0}-6_{0} \mathrm{~A}^{+}$line over the Serpens molecular core. The integration is numeric and has been done over the velocity interval -25 to $+25 \mathrm{~km} \mathrm{~s}^{-1}$ with respect to $v_{\mathrm{lsr}}=8.0 \mathrm{~km} \mathrm{~s}^{-1}$. The four Class 0 objects, Serpens SMM1, SMM3, SMM4 and S68N (labelled in Fig. 1) are clearly seen. Representative spectra obtained at different positions sampling both YSOs, molecular envelopes and outflows are shown. Besides the four Class 0 objects, three distinct outflow knots are identified, SMM1-S, SMM4-W and SMM4-S. All of these bright knots coincide with outflow positions as seen through CO observations (e.g., Davis et al. 1999). Weaker structure is also seen in the map, for example an elongation of SMM1 in the east-west direction. This corresponds to the direction of a weaker CO-outflow (Davis et al. 1999). The structure around $\mathrm{S} 68 \mathrm{~N}$ is more complex with the source itself being elongated in the NW-SE direction. Around this source a compact CS outflow has been discovered at the same position angle as the elongation (Wolf-Chase et al. 1998). Weak emission is also seen around $\mathrm{S} 68 \mathrm{~N}$ in the north-south and east-west directions.

Representative spectra of the central parts of the four envelopes are shown in Fig. 2 where emission is averaged over $3 \times$ 3 pixels (corresponding to $22^{\prime \prime} .5 \times 22^{\prime \prime} .5$ or $5600 \mathrm{AU} \times 5600 \mathrm{AU}$ ). In the following, results will be presented first for the central parts of each envelope followed by results for the extended emission in each envelope. Finally results will be presented for three strong outflow knots in the map, named SMM1-S, SMM4-W and SMM4-S.

\subsection{Emission from the central part of the envelopes}

The emission lines arising from the central part of each envelope are fitted with Gaussian line profiles to obtain the integrated emission at higher accuracy. Results are tabulated in Table 1 along with the upper level energy, $E_{\text {up }}$, and line frequency. In the spectra presented in Fig. 2 the $3 \sigma$ level is a factor of three lower, since they are each the average of nine spectra. Low- $K$ lines up to $K=3$ are clearly detected, but high- $K$ lines are not seen. This is in contrast to other low-mass YSOs with similar luminosities and/or distances and evolutionary stages, such as IRAS 16293-2422 (e.g. van Dishoeck et al. 1995) and NGC 1333 IRAS2A (Maret et al. 2005).

The integrated intensities are used to make rotational diagrams for each of the four objects. These are shown in Fig. 3.

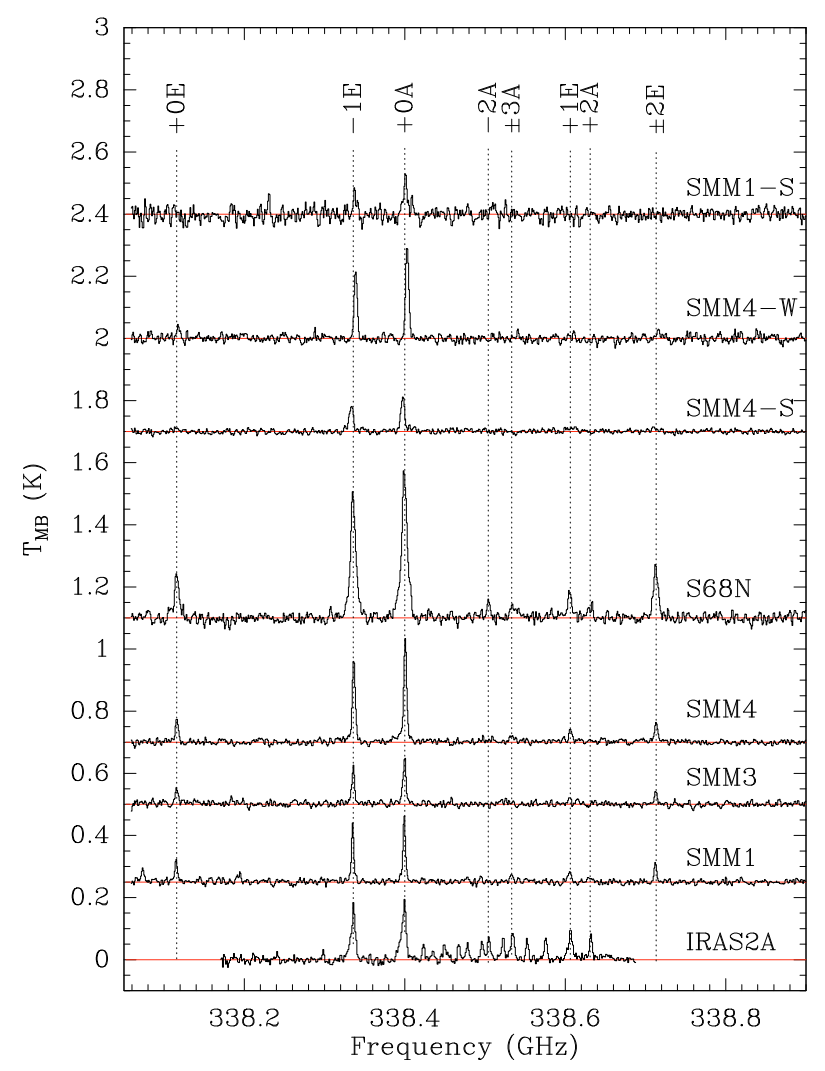

Fig. 2. Spectra of Serpens SMM1, 3, 4 and S68N. The NGC 1333IRAS2A spectrum is shown for comparison (Maret et al. 2005). Spectra at the three outflow positions, SMM1-S, SMM4-W and SMM4-S, are also shown. Lines identified in the $\mathrm{CH}_{3} \mathrm{OH} 7_{K}-6_{K}$ band are marked. Spectra were obtained by averaging emission from the peak pixel with its eight neighbouring pixels, i.e., in a box of $22^{\prime \prime} .5 \times 22^{\prime \prime}$. 5 . The SMM1, 3,4 and $\mathrm{S} 68 \mathrm{~N}$ spectra are shifted by $+0.25,0.5,0.7$ and $1.1 \mathrm{~K}$, respectively, while SMM1-S, SMM4-W and SMM4-S are shifted by 2.4, 2.0 and $1.7 \mathrm{~K}$.

Within the uncertainty, the logarithm of the derived upper level column densities, $N$, divided by the statistical weights, $g$, fall on a straight line when plotted versus the upper level energy divided by the Boltzmann constant, $k_{\mathrm{B}}$. No difference between $\mathrm{A}$ - and E-type methanol is found, thus the abundances are equal, $x\left(\mathrm{E}-\mathrm{CH}_{3} \mathrm{OH}\right)=x\left(\mathrm{~A}-\mathrm{CH}_{3} \mathrm{OH}\right)$. In the cold-temperature limit $(T \sim 10 \mathrm{~K})$ the abundance ratio is expected to be $E / A=$ 0.69 whereas it is 1 at higher temperatures, and thus little or no difference in abundance is expected (e.g., Friberg et al. 1988). The derived rotational temperature for each of the four envelopes is low, $15-20 \mathrm{~K}$, significantly lower than the rotational temperature of $\sim 80 \mathrm{~K}$ inferred for IRAS 16293-2422 and NGC 1333 IRAS2A (van Dishoeck et al. 1995; Maret et al. 2005). The estimated column densities are all of the order of $\sim 10^{14}-10^{15} \mathrm{~cm}^{-2}$. Results are tabulated in Table 2. Rotational temperatures and column densities are similar to those found by Maret et al. (2005) and Jørgensen et al. (2005b) for a larger sample of low-mass, Class 0 objects, with the exception of the two sources mentioned above.

\subsection{Extended emission from the envelopes}

To quantify the spatial distribution of methanol in each molecular envelope, the radial distribution of emission from the five strongest lines is plotted in Fig. 6. Methanol emission is extended in all envelopes, and the FWHM of emission is around 
L. E. Kristensen et al.: Methanol maps of low-mass protostellar systems. I.

Table 1. Integrated line intensities, $\int T_{\mathrm{MB}} \mathrm{d} v\left(\mathrm{~K} \mathrm{~km} \mathrm{~s}^{-1}\right)$ over the central $22^{\prime \prime} .5 \times 22^{\prime \prime} .5$ of each source ${ }^{a}$ and emission-weighted, average line-widths.

\begin{tabular}{lrrrrrrrrr}
\hline \hline Transition & $v(\mathrm{GHz})^{b}$ & $E_{\text {up }}(\mathrm{K})^{b}$ & SMM1 & SMM3 & SMM4 & S68N & SMM1-S & SMM4-W & SMM4-S \\
\hline$+0 \mathrm{E}$ & 338.1245 & 78.1 & 0.25 & 0.22 & 0.30 & 0.85 & $\ldots$ & 0.09 & 0.41 \\
$-1 \mathrm{E}$ & 338.3446 & 70.5 & 0.58 & 0.45 & 1.09 & 2.90 & 0.52 & 0.90 & 1.38 \\
$+0 \mathrm{~A}$ & 338.4087 & 65.0 & 0.71 & 0.62 & 1.35 & 3.66 & 1.06 & 1.26 & 2.21 \\
$-2 \mathrm{~A}$ & 338.5129 & 102.7 & 0.06 & 0.01 & $\ldots$ & 0.24 & 0.08 & 0.20 & $\ldots$ \\
$\pm 3 \mathrm{~A}^{c}$ & 338.5420 & 114.8 & 0.11 & 0.16 & 0.15 & 0.40 & $\ldots$ & $\ldots$ & $\ldots$ \\
$+1 \mathrm{E}$ & 338.6150 & 86.1 & 0.16 & 0.08 & 0.18 & 0.45 & $\ldots$ & 0.34 & $\ldots$ \\
$+2 \mathrm{~A}$ & 338.6399 & 102.7 & 0.09 & 0.07 & $\ldots$ & 0.26 & $\ldots$ & $\ldots$ & $\ldots$ \\
$\pm 2 \mathrm{E}^{c}$ & 338.7223 & 89.1 & 0.21 & 0.16 & 0.28 & 1.05 & $\ldots$ & 0.13 & 0.11 \\
$\Delta v\left(\mathrm{~km} \mathrm{~s}^{-1}\right)$ & & & $3.4 \pm 0.9$ & $4.4 \pm 2.5$ & $4.0 \pm 0.7$ & $7.2 \pm 1.3$ & $4.3 \pm 0.9$ & $4.0 \pm 0.3$ & $4.1 \pm 0.3$ \\
\hline
\end{tabular}

Notes. ${ }^{(a)}$ Non-detections are marked by “...”, for which an upper limit of $0.03 \mathrm{~K} \mathrm{~km} \mathrm{~s}^{-1}$ applies. ${ }^{(b)}$ Frequencies and upper level energies are from the Cologne Database for Molecular Spectroscopy (CDMS; Müller et al. 2001). ${ }^{(c)}$ Lines are blended. The frequencies and upper level energies are average values.

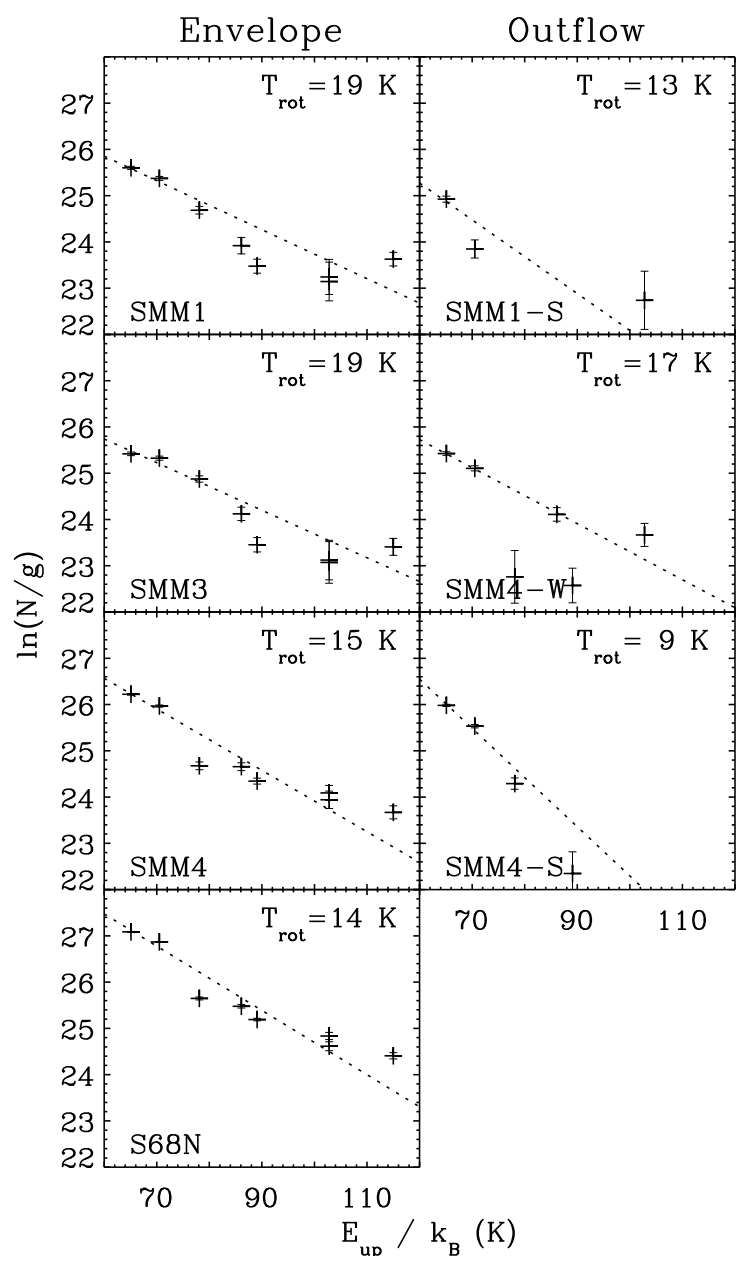

Fig. 3. Rotational diagrams with data obtained from the four spectra shown in Fig. 2. The best-fit straight line is shown in each case as a dotted line. Error bars are for $1 \sigma$ uncertainties.

2-3 times the 15" beam. Extended methanol has previously been reported in the envelope of the isolated Class 0 object L483 (Tafalla et al. 2000) in the $2_{0}-1_{0} \mathrm{~A}^{+}$and $2_{-1}-1_{-1} \mathrm{E}$ lines $\left(E_{\text {up }}=12-20 \mathrm{~K}\right)$, where it was found that the methanol emission traces total gas column density. To verify whether this is true for the Serpens envelopes, it is necessary to quantify the physical structure of the envelopes. This is done through a combination of dust continuum emission at $850 \mu \mathrm{m}$ as observed with SCUBA on the JCMT and modelling of the physical structure.
Table 2. Rotational diagram results for the observed methanol emission.

\begin{tabular}{lrr}
\hline \hline Source & $T_{\text {rot }}(\mathrm{K})$ & $N\left(10^{14} \mathrm{~cm}^{-2}\right)^{a}$ \\
\hline SMM1 & $18.9 \pm 0.9$ & $4.1 \pm 0.7$ \\
SMM3 & $19.5 \pm 1.1$ & $3.4 \pm 0.7$ \\
SMM4 & $15.1 \pm 0.4$ & $12 \pm 1.4$ \\
S68N & $14.4 \pm 0.2$ & $34 \pm 1.7$ \\
SMM1-S & $12.6 \pm 2.4$ & $5.7_{-5.7}^{+5.8}$ \\
SMM4-W & $16.5 \pm 1.3$ & $4.4 \pm 1.4$ \\
SMM4-S & $9.3 \pm 1.0$ & $34 \pm 27$ \\
\hline
\end{tabular}

Notes. ${ }^{(a)}$ Column density is the sum of A- and E-type methanol column densities.

To obtain the absolute abundances, three different methods are used. First, the $\mathrm{CH}_{3} \mathrm{OH}$ column density obtained from rotational diagrams is compared to the column density obtained from dust emission at $850 \mu \mathrm{m}$ in the same beam. Second, the $\mathrm{CH}_{3} \mathrm{OH}$ column density from rotational diagrams is compared to the column density predicted from a physical model of each source. Third, a radiative transfer model is compared directly to the observed spectrum to constrain the abundance. Results are listed in Table 4.

\subsubsection{Dust continuum emission}

It is possible to directly estimate the average column density over the central area of each envelope by assuming that the dust emission is optically thin, thermal emission at a single dust temperature, $T_{\mathrm{d}}$ :

$$
\begin{aligned}
\bar{N} & =\frac{S_{v} d^{2}}{\kappa_{v} B_{v}\left(T_{\mathrm{d}}\right) A} \\
& =4.90 \times 10^{16} \mathrm{~cm}^{-2} S_{v}(\mathrm{Jy}) d(\mathrm{pc})^{2}\left[\exp \left(\frac{16.9 \mathrm{~K}}{T_{\mathrm{d}}}\right)-1\right]
\end{aligned}
$$

Here $S_{v}$ is the continuum flux integrated over $22^{\prime \prime} .5 \times 22^{\prime \prime} .5$ at $850 \mu \mathrm{m}, d$ the distance to the object, $\kappa_{v}$ the dust opacity at $850 \mu \mathrm{m}$ taken from Ossenkopf \& Henning (1994), $B_{v}$ the Planck function at a dust temperature $T_{\mathrm{d}}$ and $A$ the total surface area, in this case $7.1 \times 10^{33} \mathrm{~cm}^{2}$. Finally a gas/dust ratio of 100 is assumed.

Reduced data from the SCUBA Legacy archive have been used (Di Francesco et al. 2008) to estimate the integrated continuum flux at $850 \mu \mathrm{m}$. An average dust temperature of $20 \mathrm{~K}$ was used following global estimates of the dust temperature over the entire Serpens molecular core of $20 \mathrm{~K}$ from Schnee et al. (2005). 
Table 3. Best fit DUSTY model input parameters and results for the physical structure of the four molecular envelopes in Serpens.

\begin{tabular}{lrrrrr}
\hline \hline Property & SMM1 & SMM3 & SMM4 & S68N & IRAS2A \\
\hline$L\left(L_{\odot}\right)$ & 37 & 2.0 & 6.3 & 3.6 & 21 \\
$d(\mathrm{pc})$ & & 230 & & & 250 \\
$p$ & 2.1 & 1.6 & 1.8 & 1.8 & 1.9 \\
$\tau(100 \mu \mathrm{m})$ & 3.8 & 1.1 & 3.0 & 3.4 & 1.8 \\
$Y\left(=r_{\text {in }} / r_{\text {out }}\right)$ & 400 & 1000 & 1000 & 800 & 500 \\
$r_{\text {in }}(\mathrm{AUU})$ & 49 & 14 & 17 & 18 & 31.3 \\
$r_{\text {out }}\left(10^{4} \mathrm{AU}\right)$ & 2.0 & 1.4 & 1.7 & 1.4 & 1.6 \\
$n_{\text {in }}\left(10^{9} \mathrm{~cm}^{-3}\right)$ & 1.7 & 0.94 & 2.8 & 3.0 & 1.0 \\
$n_{1000 \mathrm{AU}}\left(10^{6} \mathrm{~cm}^{-3}\right)$ & 3.0 & 1.0 & 1.9 & 2.2 & 1.4 \\
$N\left(\mathrm{H}_{2}\right)\left(10^{24} \mathrm{~cm}^{-2}\right)$ & 1.1 & 0.33 & 0.89 & 1.0 & 0.53 \\
Envelope mass, $T>10 \mathrm{~K}\left(M_{\odot}\right)$ & 4.1 & 2.5 & 3.9 & 3.6 & 2.2 \\
\hline
\end{tabular}

If the temperature changes to $10 \mathrm{~K}$, the column density estimate is increased by a factor of 3.3 , while if it is $30 \mathrm{~K}$, the column density is decreased by a factor of 1.8 .

By using a dust temperature of $20 \mathrm{~K}$, the average column density over a $22^{\prime \prime} .5 \times 22^{\prime \prime} .5$ region is in the range of $1-$ $4 \times 10^{23} \mathrm{~cm}^{-2}$. This leads to $\mathrm{CH}_{3} \mathrm{OH}$ fractional abundances in the range of $1-20 \times 10^{-9}$ with respect to $\mathrm{H}_{2}$, see below.

\subsubsection{DUSTY modelling of envelope properties}

Since the dust (and gas) temperatures change near the YSOs, the $\mathrm{CH}_{3} \mathrm{OH}$ emission can be further quantified through modelling of the physical parameters of the envelopes. This is done following Schöier et al. (2002) and Jørgensen et al. (2002), where the dust continuum emission is modelled with a spherically symmetric envelope with a power-law density structure heated from the inside by the protostar, using the 1D code DUSTY (Ivezic \& Elitzur 1997). Dust opacities tabulated by Ossenkopf \& Henning (1994) are used for densities of $10^{6} \mathrm{~cm}^{-3}$ and thin ice mantles (corresponding to the values in their Table 1, Col. 5). The central heating source is taken to be a black-body radiating at a temperature of $5000 \mathrm{~K}$, but model results are not very sensitive to this parameter. The output from DUSTY is dimensionless, so to make an absolute calibration it is necessary to do so against the absolute luminosity and a given distance of the source. The output can be in the form of a spectral energy distribution (SED) and a radial profile of continuum emission at a user-specified wavelength.

Only dust heating at temperatures below $250 \mathrm{~K}$ is considered (corresponding to a peak wavelength of $\sim 12 \mu \mathrm{m}$ ). Thus there is a "hole" in the inner part of the envelope extending out to a radius, $r_{\text {in }}$, where no attempt is made to model the dust emission. The physical extent of the envelope is defined by the parameter $Y=r_{\text {in }} / r_{\text {out }}$. The density profile of the envelope is described by a power-law such that $n(r) \propto r^{-p}$. Three observational constraints are used following Jørgensen et al. (2002) to identify the best-fitting dust model: the SED and the spatial distribution of continuum emission as recorded by SCUBA on the JCMT at $450 \mu \mathrm{m}$ and $850 \mu \mathrm{m}$, both available through the SCUBA legacy archive (Di Francesco et al. 2008).

SEDs were assembled from the literature, using data points from MIPS on the Spitzer Space Telescope, ISO-LWS and IRAS along with ground-based single-dish continuum measurements at sub-mm, $\mathrm{mm}$ and $\mathrm{cm}$ wavelengths. For this work, the SED database available at http://astro.kent.ac.uk/ protostars / proved invaluable (Froebrich 2005). A full table of SED points is included in Appendix A.

The reduced SCUBA continuum emission maps from the SCUBA legacy archive have been smoothed to equivalent beam sizes of $11^{\prime \prime}$ and 19.5 at 450 and $850 \mu \mathrm{m}$, respectively (Di Francesco et al. 2008). Along with emission maps, maps containing the error on each pixel are also provided, facilitating error analysis. The radial profile of each object was compiled by considering the emission in annuli extending from the pixel containing the maximum value of emission. The maps were carefully checked, and directions containing contamination from other objects were avoided.

Three DUSTY input values were varied: the physical size of the envelope, $Y$, the opacity at $100 \mu \mathrm{m}, \tau(100 \mu \mathrm{m})$ and the powerlaw slope of the radial density profile, $p$. A small grid of simulations was calculated and the best-fit model for each envelope was found using a $\chi^{2}$ method, following Jørgensen et al. (2002). The best-fit model parameters are listed in Table 3 along with physical parameters such as the extent of the envelope, the local density at the inner radius, the column density and the total envelope mass. The best-fit model predictions are overplotted on actual data in Fig. 4.

In general agreement between the modelled and observed properties are good, except in the case of the modelled $850 \mu \mathrm{m}$ radial profile of $\mathrm{S} 68 \mathrm{~N}$. The model prediction is consistently a factor of 2-3 lower than observed values. Part of the reason is that since several sub-mm sources are present close to $\mathrm{S} 68 \mathrm{~N}$, only emission from the NW direction is modelled here, implying that the uncertainty on individual data points is higher than in the case of SMM1, SMM3 and SMM4. In this particular direction there may still be contamination, and thus emphasis is placed primarily on modelling of the SED. Moreover, the radial emission profile at $450 \mu \mathrm{m}$ is much steeper than the $850 \mu \mathrm{m}$ profile, indicating that there may be more cold, ambient cloud material toward $\mathrm{S} 68 \mathrm{~N}$. The radial profile obtained at $450 \mu \mathrm{m}$ is well reproduced by the best-fit DUSTY model.

From the DUSTY model it is possible to estimate the average column density over a region of $22^{\prime \prime} .5 \times 22$ '. 5 and compare this to the $\mathrm{CH}_{3} \mathrm{OH}$ column density. The average column density is found to be $\sim(1.0 \pm 0.2) \times 10^{23} \mathrm{~cm}^{-2}$. This leads to an abundance of $\sim 3 \times 10^{-9}$ with respect to $\mathrm{H}_{2}$ for SMM1 and SMM3 to $\sim 30 \times$ $10^{-9}$ for S68N (see Table 4 for details).

\subsubsection{RATRAN simulation}

In the following the abundance is constrained through direct radiative transfer modelling. This has been done using the code, RATRAN (Hogerheijde \& van der Tak 2000) in conjunction with molecular data from LAMDA (Schöier et al. 2005). This opportunity was used to update collisional data in the LAMDA database for methanol using newly released rate coefficients for collisions between para- $\mathrm{H}_{2}$ and $\mathrm{A}$ - and E-type methanol up to 


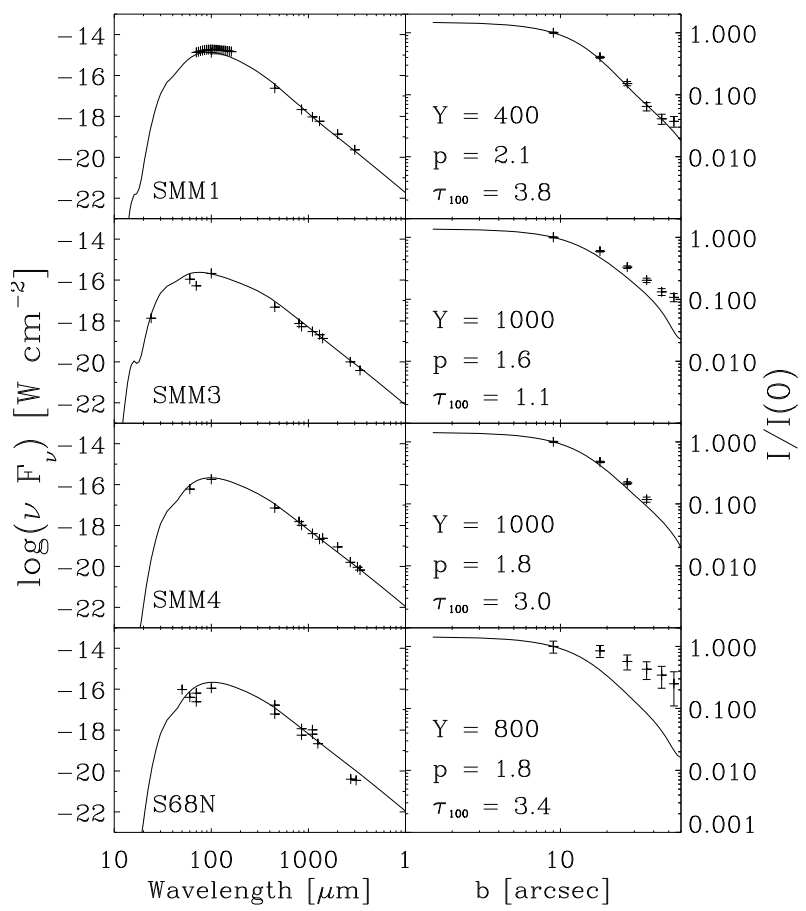

Fig. 4. DUSTY modelling results of the molecular envelopes of SMM1, 3, 4 and S68N. The left panel shows the SEDs of all four objects, whereas the right panel shows the normalized radial dust emission profile as obtained with SCUBA (Di Francesco et al. 2008). Best-fit results are over-plotted as solid lines, and the values for the best-fit models are given in the right-hand panel.

and including $J=9$, corresponding to an upper level energy of $\sim 500 \mathrm{~K}$ (Pottage et al. 2004). The rate coefficients are given for temperatures from 5 to $200 \mathrm{~K}$. The coefficients are not complete up to upper level energies of $500 \mathrm{~K}$ since they are limited to $J<10$. In the case of A-type methanol with $K=0$, this corresponds to an upper level energy of $\sim 100 \mathrm{~K}$. Data have been extrapolated so that they are complete up to an upper level energy of $385 \mathrm{~K}$ corresponding to the first torsional state. For the extrapolation only transitions between $K$-ladders and $\Delta J=1-5$ are considered. The collisional rate coefficients are found to be proportional to $\sqrt{T} / \Delta J$ which is used for the extrapolation (Leurini et al. 2007). Level energies and Einstein $A$-values are taken from the Cologne Database for Molecular Spectroscopy (CDMS; Müller et al. 2001).

The density and temperature profile of the DUSTY modelling is used for the physical structure of the envelope. No effort is made to model the line widths, but instead a constant, turbulent line width of $3.0-5.0 \mathrm{~km} \mathrm{~s}^{-1}$ is assumed, depending on source. This is not a measure of the actual turbulence in the region, but only a means to ensure that the modelled linewidth corresponds to the observed. For each source a small grid of RATRAN models has been run with a so-called "jump" abundance structure. The jump was located at $T_{\text {dust }}=80 \mathrm{~K}(R=50-125 \mathrm{AU})$ with an increase in the abundance of a factor of $1-10^{3}$ corresponding to most $\mathrm{CH}_{3} \mathrm{OH}$ evaporating off of the grains. When running the RATRAN models, great care has been taken to ensure that the central region containing the jump is properly sampled. In order to do this the pixel-size is set to 0.2 (45 AU) and the central pixels were oversampled by a factor of 50 .
A $\chi^{2}$ method is then used to determine the best-fit model integrated emission compared to observed integrated emission. This is done separately for A- and E-type $\mathrm{CH}_{3} \mathrm{OH}$ to examine relative abundances. No significant difference is found between A- and E-type $\mathrm{CH}_{3} \mathrm{OH}$ abundances, thus confirming the results obtained from the rotational diagrams. All abundances are listed in Table 4. Diagrams illustrating the variation of $\chi^{2}$ with inner and outer abundance are shown in Fig. 5. Here it may be seen that the inner abundance is not well constrained, and it is only possible to provide upper limits ranging from $2 \times 10^{-9}$ (SMM1) to $5 \times 10^{-7}$ (SMM3 and S68N). On the other hand, the outer envelope abundance is very well constrained for all sources and lies in the range of $10^{-9}$ (SMM1) to greater than $10^{-8}(\mathrm{~S} 68 \mathrm{~N})$. The methanol enhancements, $x_{\text {in }} / x_{\text {out }}$, are from $\lesssim 2-3$ (SMM1) to $\lesssim 200$ (SMM3). Due to the lack of high- $K$ lines it is not possible to further constrain the inner abundance.

\subsubsection{Comparison of abundance measurements}

The three methods for determining the abundance may be divided into the following categories:

1. Direct observational abundance assuming LTE in terms of $\mathrm{CH}_{3} \mathrm{OH}$ excitation and optically thin dust emission.

2. Combined direct and model abundance, still assuming LTE in terms of $\mathrm{CH}_{3} \mathrm{OH}$ excitation but modelling the dust emission with a range of temperatures.

3. Modelling of the $\mathrm{CH}_{3} \mathrm{OH}$ emission within a physical, spherically symmetric model with varying dust and gas temperatures and densities.

Despite the obvious differences and levels of complexity between the three methods, the results in terms of average methanol abundances are remarkably similar. Typically, variations are within a factor of 2-3.

In the following only results from the RATRAN modelling will be discussed. Since this method takes the full density and temperature structure into account it will be the more accurate of the three as demonstrated for other molecules (e.g., Hogerheijde \& van der Tak 2000; Jørgensen et al. 2002, 2004).

\subsection{Outer envelope abundance structure}

In Fig. 6 the column densities as predicted by the DUSTY modelling and the $850 \mu \mathrm{m}$ dust continuum emission radial profiles are overlaid. The methanol emission profiles follow both the dust emission and column density profiles very closely over scales from $\sim 5500 \mathrm{AU}$ to greater than 12000 AU. Because the distribution is independent of rotational line, the rotational temperature (corresponding to line ratio) is constant throughout the envelope and the methanol column density scales directly with the envelope column density, similar to the case of L483 (Tafalla et al. 2000). Thus, over this range of radial distances, the average $\mathrm{CH}_{3} \mathrm{OH}$ gas abundance is constant. In the case of $\mathrm{S} 68 \mathrm{~N}$ the agreement between the observed and simulated radial profiles is not good since the simulation under-estimates the emission at larger radii. Furthermore, the outer envelope abundance as derived from rotational diagrams or from comparison with dust emission agrees to within a factor of 2 with the outer abundance as derived from the RATRAN modelling.

\subsection{Emission from outflow positions}

Methanol is detected along the outflows throughout the emission map (Fig. 1). In the following, focus will be placed on 


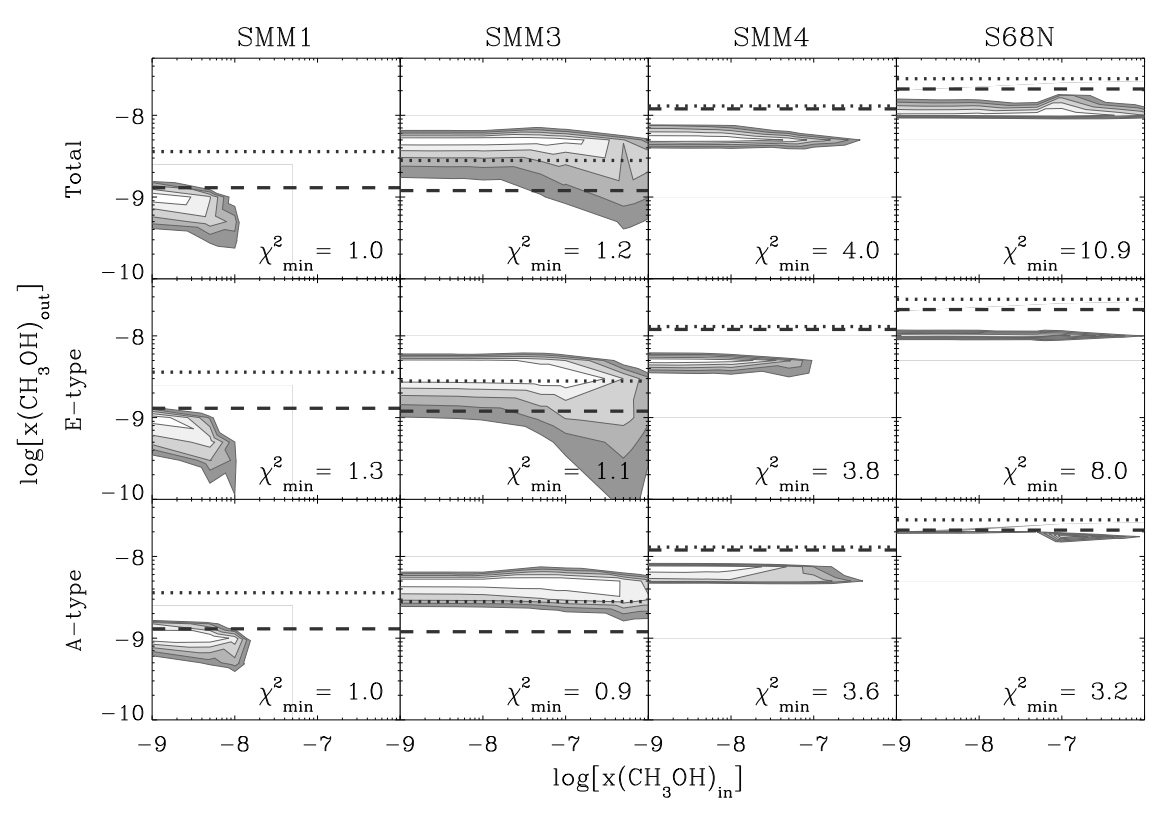

Fig. 5. Reduced $\chi^{2}$ distribution as a function of inner and outer abundance for A- and E-type $\mathrm{CH}_{3} \mathrm{OH}$ as well as the combined value. Contours are for $1,2,3,4$ and $5 \sigma$. The dotted line corresponds to the abundance derived using $N\left(\mathrm{H}_{2}\right)$ from DUSTY modelling, and the dashed line is the abundance derived using $N\left(\mathrm{H}_{2}\right)$ directly from SCUBA dust continuum emission. The minimum $\chi_{\text {red }}^{2}$ is given in each panel.

Table 4. Average total gas column densities and fractional abundances of $\mathrm{CH}_{3} \mathrm{OH}$.

\begin{tabular}{lrrrrrr}
\hline \hline Source & $\begin{array}{r}N_{\text {DUSTY }}^{a} \\
\left.\mathrm{~cm}^{-2}\right)\end{array}$ & $\begin{array}{r}N_{\text {SCUBA }}^{b} \\
\left(10^{23} \mathrm{~cm}^{-2}\right)\end{array}$ & $\begin{array}{r}x\left(\mathrm{CH}_{3} \mathrm{OH}\right)_{\text {DUSTY }}^{c} \\
\times 10^{-9}\end{array}$ & $\begin{array}{r}x\left(\mathrm{CH}_{3} \mathrm{OH}\right)_{\text {SCUBA }}^{c} \\
\times 10^{-9}\end{array}$ & $\begin{array}{r}x\left(\mathrm{CH}_{3} \mathrm{OH}\right)_{\text {out }}^{c, d} \\
\times 10^{-9}\end{array}$ & $\begin{array}{r}x\left(\mathrm{CH} \mathrm{H}_{3} \mathrm{OH}\right)_{\text {in }}^{c, d} \\
\times 10^{-8}\end{array}$ \\
\hline SMM1 & 1.3 & $3.5 \pm 0.3$ & $3.6 \pm 0.9$ & $1.3 \pm 0.4$ & 1 & $\leq 0.3$ \\
SMM3 & 0.83 & $2.0 \pm 0.13$ & $2.8 \pm 0.7$ & $1.2 \pm 0.3$ & 4 & $\leq 50$ \\
SMM4 & 1.1 & $1.2 \pm 0.07$ & $13 \pm 3$ & $12 \pm 3$ & 5 & $\leq 5$ \\
S68N & 1.2 & $1.7 \pm 0.10$ & $28 \pm 2$ & $21 \pm 2$ & 10 & $\leq 40$ \\
\hline
\end{tabular}

Notes. ${ }^{(a)}$ Gas column density, $N\left(\mathrm{H}_{2}\right)$, obtained from DUSTY modelling over a region of $22^{\prime \prime} 5 \times 22^{\prime \prime} .5$ assuming a gas:dust ratio of $100 .{ }^{(b)}$ Gas column density, $N\left(\mathrm{H}_{2}\right)$,obtained from SCUBA emission at $850 \mu \mathrm{m}$ assuming a constant dust temperature of $20 \mathrm{~K}^{(c)}$ Fractional $\mathrm{CH}_{3} \mathrm{OH}$ abundance summed over A- and E-type $\mathrm{CH}_{3} \mathrm{OH} .{ }^{(d)}$ Inner and outer abundance in the RATRAN jump-model.

emission from three distinct knots labelled SMM1-S, SMM4-W and SMM4-S in Fig. 1. The elongated emission associated with $\mathrm{S} 68 \mathrm{~N}$ indicates that a compact shock is responsible for sputtering $\mathrm{CH}_{3} \mathrm{OH}$ from the grain mantles into the gas phase. This outflow has already been observed in CS $J=3-2$ emission (Wolf-Chase et al. 1998). The line profiles associated with this outflow are symmetric and Gaussian in nature making an accurate disentanglement of the contributions from envelope and outflow difficult. Unlike the above mentioned outflow knots, emission is spatially coincident with the source position. For this reason the S68N outflow is not included in the following discussion.

To investigate the properties of each outflow knot, spectra are extracted from the map and rotational diagrams are made in the same manner as above (see Figs. 2 and 3). The intensity of the strong $77_{0}-6_{0} \mathrm{~A}^{+}$line is comparable to the emission from SMM4 in all three knots. The detected transitions and associated intensities are listed in Table 1 . The lines are shifted by $\sim 1 \mathrm{~km} \mathrm{~s}^{-1}$ with respect to each other and with respect to the source velocities. Line profiles are symmetric and Gaussian in nature, with no obvious line-wings. Nevertheless, methanol clearly traces the outflow activity associated with SMM1, SMM4 and S68N as seen in Fig. 7, whereas little emission is associated with SMM3. In Fig. 7 the contributions from red- and blue-shifted emission are estimated by integrating over the velocity intervals from -5 to $7 \mathrm{~km} \mathrm{~s}^{-1}$ and 10 to $20 \mathrm{~km} \mathrm{~s}^{-1}$, respectively. This is to be compared with the average source velocity, $v_{\mathrm{lsr}}=8.5 \mathrm{~km} \mathrm{~s}^{-1}$.

Results obtained from the rotational diagrams are tabulated in Table 2. The rotational temperature is $\sim 10-15 \mathrm{~K}$, indicating sub-thermal excitation at all three outflow positions, as found typically also in other outflows because of the relatively low density compared with the central envelope (e.g., Bachiller et al. 1995). The column densities range from a few $\times 10^{14} \mathrm{~cm}^{-2}$ (SMM1-S and SMM4-W) to $\sim 3 \times 10^{15} \mathrm{~cm}^{-2}$ (SMM4-S). To obtain accurate abundance estimates of methanol in shocked regions, it is again imperative to estimate the gas column density accurately. In this case ${ }^{12} \mathrm{CO} J=3-2$ data from the JCMT science archive, obtained in the context of the JCMT Gould Belt legacy survey, are used (Graves et al. 2010). The principle for obtaining the column density from ${ }^{12} \mathrm{CO}$ in the shocked gas is that emission from the higher velocity line-wings is optically thin. This assumption has been shown to be valid for a number of molecular outflows (see e.g., Bachiller \& Tafalla 1999, for a review). In Fig. 8 the line profiles are shown for the three outflow positions analysed here. Emission from SMM1-S and SMM4-W is blue-shifted, while emission from SMM4-S is red-shifted. The CO line-wings are not as pronounced as in other well-known outflow sources (e.g., Blake et al. 1995), but are slightly shifted (up to $\pm 5 \mathrm{~km} \mathrm{~s}^{-1}$ ) with respect to a $v_{\mathrm{lsr}}$ of $8.5 \mathrm{~km} \mathrm{~s}^{-1}$. This indicates that the flows may be caused by J-type shocks rather than C-type shocks (Hollenbach 1997). Molecular emission is expected to have a Gaussian velocity profile around the shock velocity in a J-type shock, whereas emission caused by C-type shocks is expected to show a "classic", triangular line profile. However, since the observed lines are only shifted by $\pm 5 \mathrm{~km} \mathrm{~s}^{-1}$ at most, so the J-type shock speed would be lower than this. $\mathrm{CH}_{3} \mathrm{OH}$ is also efficiently destroyed in the sputtering process in J-type shocks at velocities greater than $10 \mathrm{~km} \mathrm{~s}^{-1}$. The actual 
L. E. Kristensen et al.: Methanol maps of low-mass protostellar systems. I.

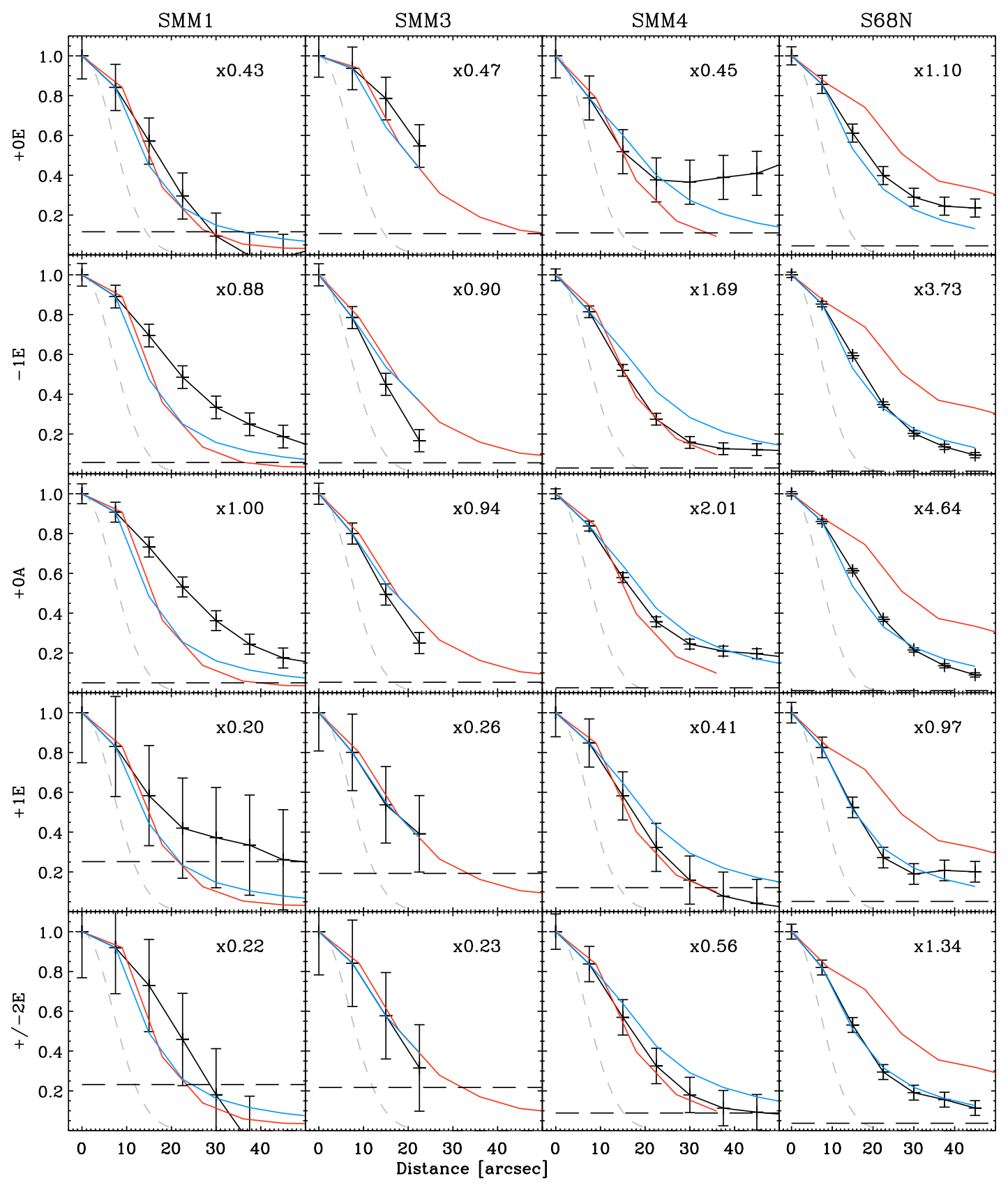

Fig. 6. Spatial distribution of methanol emission in the four objects, Serpens SMM1, 3, 4 and S68N. The integrated emission of the five lines 7-6 $+0 \mathrm{E},-1 \mathrm{E},+0 \mathrm{~A},+1 \mathrm{E}$ and $\pm 2 \mathrm{E}$ is shown (black). The error-bars are for $1 \sigma$ uncertainties. Overlaid is the dust continuum emission as obtained from SCUBA (red) and the DUSTY dust continuum modeling column density (blue). All profiles have been normalized to 1. The normalization factor for the peak emission is shown in each diagram. The $3 \sigma$ noise level is shown as a dashed line, and the grey, dashed line shows the beam profile.

$\mathrm{CH}_{3} \mathrm{OH}$ enhancement may be lower in the outflows in Serpens compared to e.g., L1157, something which is also discussed for the case of NGC 2071 (Garay et al. 2000). Another possibility is that the flows are C-type shocks moving very close to the plane of the sky, in which case the line-wings will not be prominent. This is suported by $\mathrm{SiO} J=2-1$ observations of SMM4, where the lines were also found to be narrow (FWHM $\sim 5 \mathrm{~km} \mathrm{~s}^{-1}$; Garay et al. 2002). With the present data-set it is not possible to differentiate the two scenarios.

Emission was integrated over the velocity intervals given in Davis et al. (1999). From this the CO column density is calculated under the assumption that emission is optically thin and that the gas temperature is $100 \mathrm{~K}$. The calculation of the column density is insensitive to the choice of temperature, as long as it is in the interval of $\sim 50-150 \mathrm{~K}$. The calculation is more sensitive to the choice of velocity interval over which $\mathrm{CO}$ emission is integrated, as this may vary by up to a factor of 2 , when changing the velocity interval with $1 \mathrm{~km} \mathrm{~s}^{-1}$. The column density is then converted to total gas column density using a standard abundance ratio of $\mathrm{CO}: \mathrm{H}_{2}$ of $10^{-4}: 1$ and results are compared to $\mathrm{CH}_{3} \mathrm{OH}$ column densities to obtain an abundance. The integrated $\mathrm{CO}$ intensities, column densities and $\mathrm{CH}_{3} \mathrm{OH}$ abundances are all tabulated in Table 5. The gas column density in all three outflow positions is $\sim 10^{20} \mathrm{~cm}^{-2}$ resulting in abundances 


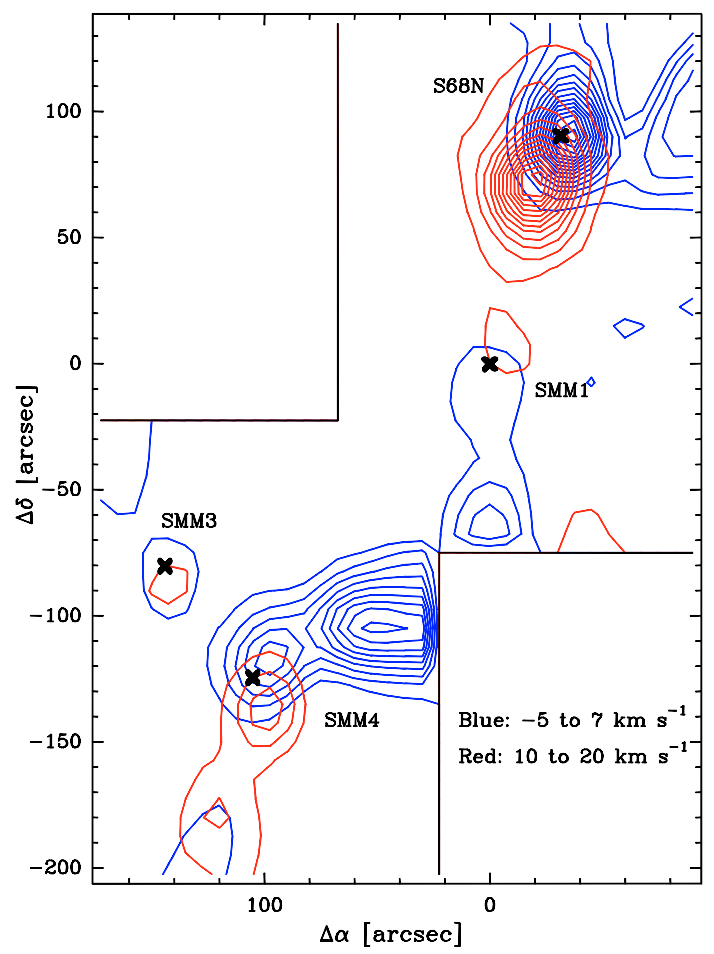

Fig. 7. Outflow emission in Serpens as traced by the $\mathrm{CH}_{3} \mathrm{OH} 7_{0}-6_{0} \mathrm{~A}^{+}$ line. Contours are for red- and blue-shifted outflow emission and are at $3 \sigma, 6 \sigma, 9 \sigma, \ldots$ Crosses mark the positions of SMM1, SMM3, SMM4 and $\mathrm{S} 68 \mathrm{~N}$.

of $\sim 1-10 \times 10^{-6}$ with respect to $\mathrm{H}_{2}$. Due to the uncertainties on the derived $\mathrm{CH}_{3} \mathrm{OH}$ column densities and on the gas column density, the abundances should be seen as order of magnitude estimates. Compared to the envelope abundances of SMM1 and SMM4, this translates to methanol enhancements in the outflows of $z 10^{3}$. This is similar to what is found in other outflows; for example, the enhancement for the L1157 blue lobe is of the order of 400 (Bachiller et al. 1995; Bachiller \& Pérez Gutiérrez 1997). It should be noted, however, that the methanol line profiles are highly asymetric in the L1157 outflow as are the CO line profiles.

$\mathrm{C}^{18} \mathrm{O}$ is often used as a tracer of quiescent gas column density. If $\mathrm{C}^{18} \mathrm{O} J=3-2$ emission is used rather than ${ }^{12} \mathrm{CO}$ emission as above, the gas column density is increased by $\sim$ two orders of magnitude to $\sim 10^{22} \mathrm{~cm}^{-2}$. This leads to a decrease in $\mathrm{CH}_{3} \mathrm{OH}$ abundance and therefore also a decrease in the enhancement. Representing the other extreme, typical enhancements are of the order of 5-50 (see Table 5). Ideally, emission should be integrated over the same velocity interval for comparison, but in this case, part of the $\mathrm{CH}_{3} \mathrm{OH}$ line overlaps with the ${ }^{12} \mathrm{CO}$ profile, part of it overlaps with the $\mathrm{C}^{18} \mathrm{O}$ profile, which is why these two abundance measurements should be seen as extreme values.

\section{Discussion}

The inferred abundances are all larger than what can be produced by pure gas-phase reactions (e.g. Garrod et al. 2006). Thus the observed $\mathrm{CH}_{3} \mathrm{OH}$ gas must be formed on grains and subsequently have desorbed. In the following, possible scenarios for methanol desorption and excitation are discussed. Finally speculations on the nature of the individual young stellar objects in Serpens are made.

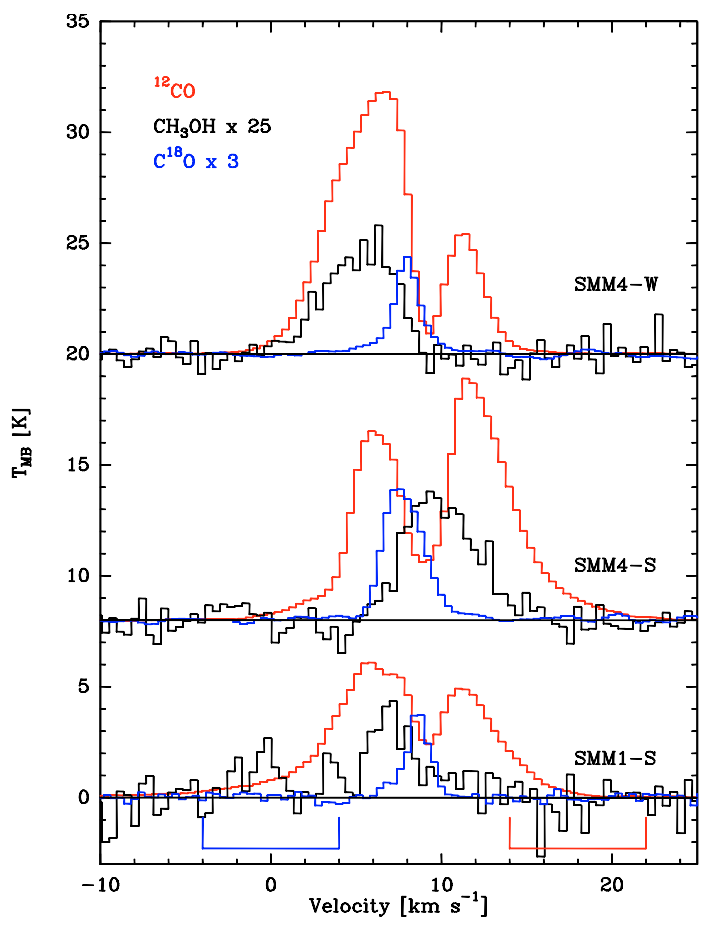

Fig. 8. Comparison of outflow line profiles. For each outflow position, SMM1-S, SMM4-W and SMM4-S, line profiles of ${ }^{12} \mathrm{CO} J=3-2$ (red), $\mathrm{C}^{18} \mathrm{O} \mathrm{J}=3-2$ (blue) and $\mathrm{CH}_{3} \mathrm{OH} 7_{0}-6_{0} \mathrm{~A}^{+}$(black) are shown. $\mathrm{C}^{18} \mathrm{O}$ and $\mathrm{CH}_{3} \mathrm{OH}$ spectra have been multiplied by 3 and 25, respectively, for clarity. SMM4-S and SMM4-W spectra have been shifted by 8 and $20 \mathrm{~K}$. The masks at the bottom indicate the velocity range over which ${ }^{12} \mathrm{CO}$ emission is integrated to obtain the outflow column density.

\section{1. $\mathrm{CH}_{3} \mathrm{OH}$ grain surface formation}

Methanol is formed on the surfaces of interstellar dust grains through hydrogenation of $\mathrm{CO}$, a mechanism which has been studied in detail in the laboratory and in theoretical models of surface chemistry (Hiraoka et al. 2002; Watanabe \& Kouchi 2002; Fuchs et al. 2009). To form large amounts of $\mathrm{CH}_{3} \mathrm{OH}$, it is imperative that $\mathrm{CO}$ freezes effectively out onto (waterice covered) dust grains. Toward several pre-stellar cores (e.g., B68, Bergin et al. 2002) and Class 0 objects (e.g., Jørgensen et al. 2005c) CO has been observed to freeze out very efficiently at temperatures lower than $\sim 20 \mathrm{~K}$ and densities greater than $\sim 10^{5} \mathrm{~cm}^{-3}$. This "catastrophic" CO freeze-out has been observed directly through observations of $\mathrm{CO}$ ice abundances which show an increase in the amount of solid $\mathrm{CO}$ with respect to $\mathrm{H}_{2} \mathrm{O}$ ice in the densest regions (Pontoppidan 2006). Through use of DUSTY modelling discussed above it is possible to estimate the extent of the CO freeze-out zone in each of the Serpens Class 0 objects quantitatively, which is illustrated in Fig. 9. Here the predicted density and temperature profiles are shown as a function of distance from the protostar itself. The density profiles of all four envelopes are very similar, however SMM1 stands out in terms of temperature profile. Due to the higher luminosity (30 $\left.L_{\odot}\right)$ of SMM1 with respect to the other source luminosities $\left(\sim 5 L_{\odot}\right)$, the envelope temperature is also higher throughout.

The zone over which CO freezes out in SMM1 is significantly smaller than that of the other sources, SMM3, SMM4 and S68N. In the case of SMM1, the present freeze-out zone extends from 1000-5000 AU whereas the other envelopes have freezeout zones starting at a few hundred AU and extend out to the same distance as for SMM1 (see Fig. 9). This indicates that at present $\mathrm{CO}$ is not freezing out efficiently in the SMM1 envelope, 
L. E. Kristensen et al.: Methanol maps of low-mass protostellar systems. I.

Table 5. Gas column density, $N\left(\mathrm{H}_{2}\right)$ and $\mathrm{CH}_{3} \mathrm{OH}$ abundance in outflow knots as determined from ${ }^{12} \mathrm{CO} J=3-2$ and $\mathrm{C}^{18} \mathrm{O} J=3-2$ emission.

\begin{tabular}{lrrrrrrrr}
\hline \hline & \multicolumn{4}{c}{${ }^{12} \mathrm{CO}$} & \multicolumn{3}{c}{$\mathrm{C}^{18} \mathrm{O}$} \\
Source & $\begin{array}{r}\int_{\mathrm{K} \mathrm{km} \mathrm{s}} T_{\mathrm{MB}} \mathrm{d} v \\
\left(\mathrm{Km}^{-1}\right)\end{array}$ & $\begin{array}{c}N_{\mathrm{CO}}^{\mathrm{of}} \\
\left(10^{16} \mathrm{~cm}^{-2}\right)\end{array}$ & $x\left(\mathrm{CH}_{3} \mathrm{OH}\right)$ \\
$\times 10^{-6}$ & $x_{\text {of }} / x_{\text {env }}$ & $\begin{array}{c}\int T_{\mathrm{MB}} \mathrm{d} v \\
\left(\mathrm{~K} \mathrm{~km} \mathrm{~s}^{-1}\right)\end{array}$ & $\begin{array}{r}N_{\mathrm{CO}} \\
\left(10^{18} \mathrm{~cm}^{-2}\right)\end{array}$ & $\begin{array}{r}\left.\mathrm{CH}_{3} \mathrm{OH}\right) \\
\times 10^{-8}\end{array}$ & $x_{\text {of }} / x_{\text {env }}$ \\
\hline SMM1-S & 27.5 & 1.2 & 5.1 & 5000 & 3.6 & 1.1 & 5.2 & 50 \\
SMM4-W & 40.7 & 0.9 & 5.0 & 1000 & 4.6 & 1.4 & 3.1 & 6 \\
SMM4-S & 44.7 & 1.7 & 19.6 & 3900 & 9.8 & 2.9 & 12 & 25 \\
\hline
\end{tabular}

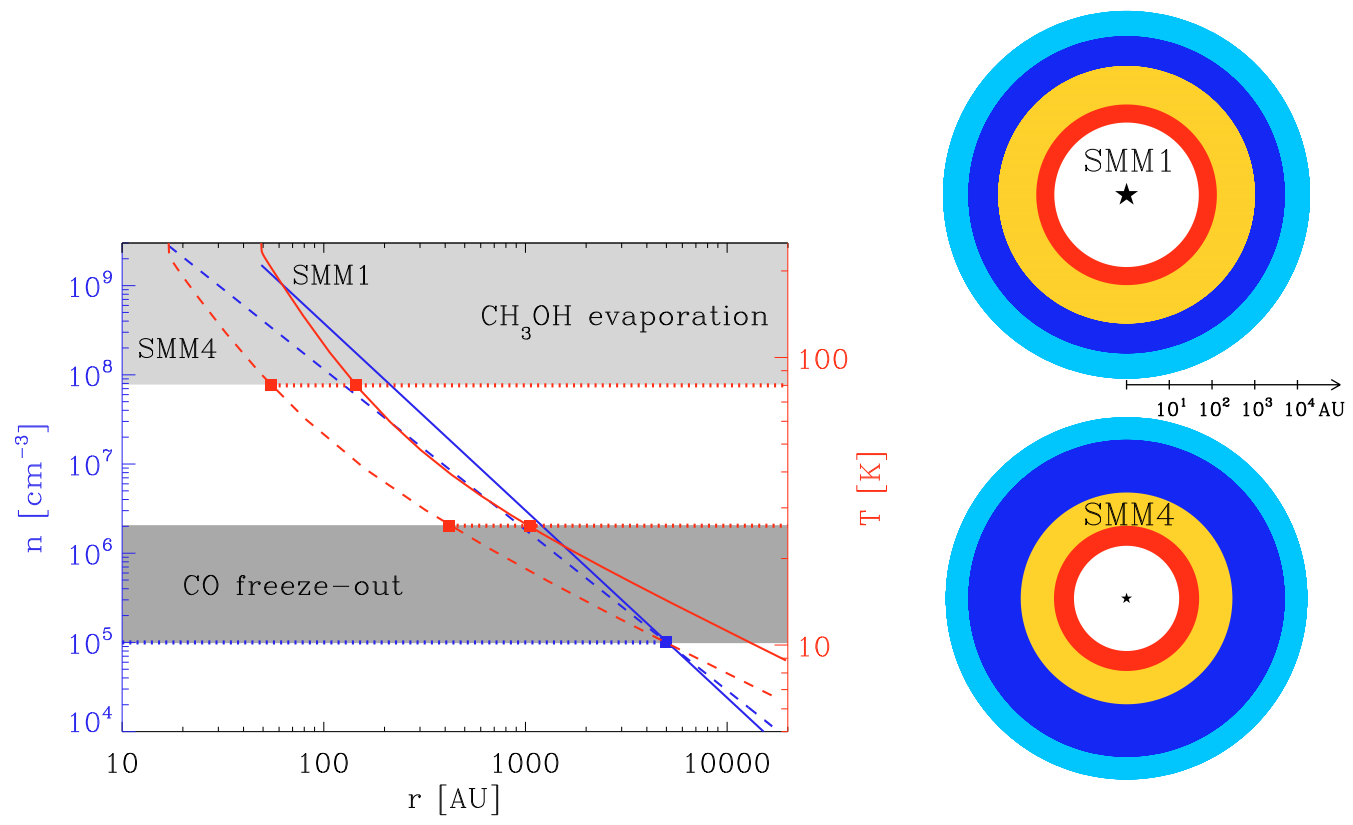

Fig. 9. Density and temperature profiles of the SMM1 and SMM4 envelopes as obtained from DUSTY modelling. The radial density profile is displayed in blue (left axis) and kinetic dust temperature in red (right axis). The profiles are shown for the SMM1 envelope (full line) and the SMM4 envelope (dashed line). The CO freeze-out zone, characterised by temperatures lower than $25 \mathrm{~K}$ and densities greater than $10^{5} \mathrm{~cm}^{-3}$, is shown in dark gray, whereas the methanol evaporation zone $(T>80 \mathrm{~K})$ is shown in light gray. To the right is a cartoon illustrating the differences between the envelopes surrounding SMM1 and SMM4. Both illustrations are to scale and the dark blue regions correspond to the CO freeze-out zones while red indicates $\mathrm{CH}_{3} \mathrm{OH}$ evaporation zones. Note the much smaller $\mathrm{CO}$ freeze-out zone for SMM1.

so any efficient methanol formation has probably ceased at this point in time. The colder envelopes surrounding SMM3, SMM4 and $\mathrm{S} 68 \mathrm{~N}$ could still be forming $\mathrm{CH}_{3} \mathrm{OH}$.

In terms of observing methanol directly in the ice itself, Pontoppidan et al. (2004) studied a small region extending south of SMM4 from $4000 \mathrm{AU}$ to $12000 \mathrm{AU}$ by observing the $3.54 \mu \mathrm{m}$ $\mathrm{CH}_{3} \mathrm{OH}$ features in absorption against background and embedded stars. The extent is illustrated by the white line in Fig. 1. In this region the $\mathrm{CH}_{3} \mathrm{OH}$-ice abundance is constant at $28 \%$ with respect to water ice or $3 \times 10^{-5}$ with respect to gas-phase $\mathrm{H}_{2}$. This corroborates the interpretation presented here, that the gas phase abundance of $\mathrm{CH}_{3} \mathrm{OH}$ is low and constant out to $12000 \mathrm{AU}$ in the SMM4 envelope. Beyond this line the $\mathrm{CH}_{3} \mathrm{OH}$-ice abundance drops by at least an order of magnitude and Pontoppidan et al. could only determine upper limits. It is interesting to note that the location where the ice abundance drops is where one of the outflow knots starts (SMM4-S; see Fig. 1). Thus the reason for the drop is a combination of the envelope being more tenuous $\left(n_{\mathrm{H}}<10^{4} \mathrm{~cm}^{-3}\right)$ far from the protostar, so that $\mathrm{CO}$ does not freeze out very efficiently, while at the same time whatever methanol is in the ice is sputtered into the gas phase by the outflow.
Cuppen et al. (2009) have recently studied the formation of $\mathrm{CH}_{3} \mathrm{OH}$ on ice surfaces using a Monte Carlo method, in which the gas-grain chemistry based on laboratory data is simulated microscopically over long time-scales. The limiting factors in producing methanol is the availability of both $\mathrm{CO}$ and $\mathrm{H}$ on the grain surfaces, and so results show that $\mathrm{CO}$ hydrogenates efficiently to form $\mathrm{CH}_{3} \mathrm{OH}$, especially at temperatures lower than $12 \mathrm{~K}$ where atomic hydrogen can be retained efficiently. After $10^{5}$ years $\mathrm{CH}_{3} \mathrm{OH}$ may form up to 100 individual mono-layers on the grain, comparable to that of water ice. Hence, the ice abundance of methanol in the outer parts of the envelope will depend strongly on the temperature. Because the envelopes of SMM3, SMM4 and S68N are significantly colder than that of SMM1, they would still be actively producing methanol, which could explain the higher gas phase abundances (see Table 4).

Observations show a different behaviour of $\mathrm{C}^{18} \mathrm{O}$ at the center position of $\mathrm{S} 68 \mathrm{~N}$ compared with the center position of SMM1. Figure 10 presents $\mathrm{C}^{18} \mathrm{O} J=3-2$ emission in colour and methanol emission from the $7_{0}-6_{0} \mathrm{~A}^{+}$line overlaid as contours. The morphology of the $\mathrm{C}^{18} \mathrm{O}$ emission shows a peak toward SMM1, as expected for a warm envelope, and a ring of emission toward $\mathrm{S} 68 \mathrm{~N}$. The $\mathrm{C}^{18} \mathrm{O}$ abundances towards the two central positions are measured to be 3 and $1 \times 10^{-8}$ respectively, 


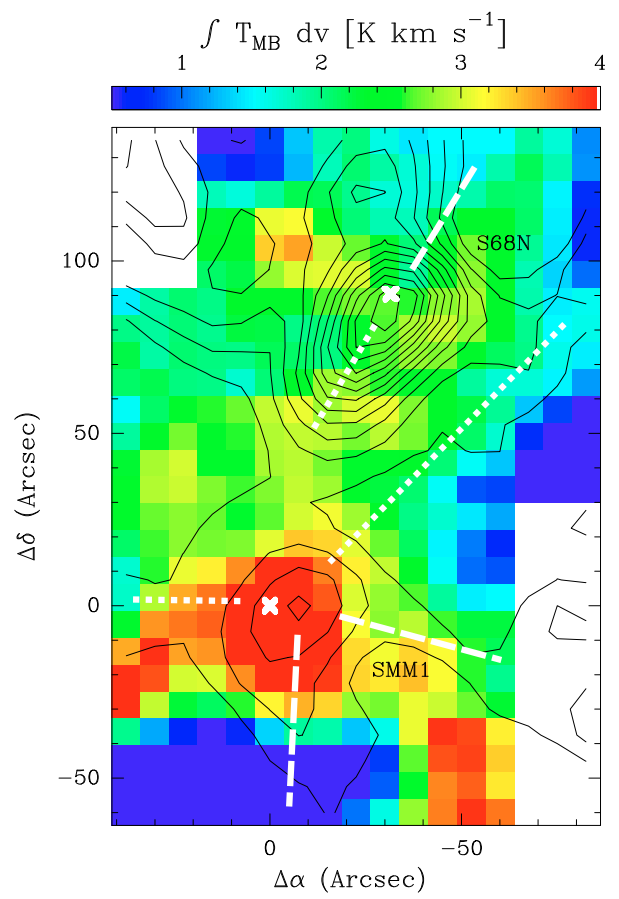

Fig. 10. Integrated $\mathrm{C}^{18} \mathrm{O}, J=3-2$ emission $\left(\int T_{\mathrm{mb}} \mathrm{d} v\right)$ is shown in colour, with integrated $\mathrm{CH}_{3} \mathrm{OH} 7_{0}-6_{0} \mathrm{~A}^{+}$emission overlaid as contours. The image is centered on Serpens SMM1 with S68N located to the north. Outflow directions are indicated by dashed (red-shifted) and dotted (blue-shifted) lines.

based on integrated $\mathrm{C}^{18} \mathrm{O}$ intensities of 4.3 and $2.4 \mathrm{~K} \mathrm{~km} \mathrm{~s}^{-1}$ and the gas column densities derived from dust continuum emission (Table 4). This is to be compared to a standard $\mathrm{C}^{18} \mathrm{O}$ abundance of $1.8 \times 10^{-7}$ for a normal abundance of $\mathrm{CO} / \mathrm{H}_{2}=10^{-4}$ and an ${ }^{16} \mathrm{O} /{ }^{18} \mathrm{O}$ ratio of 550 (Wilson \& Rood 1994). Thus, $\mathrm{CO}$ is depleted by a factor of $\sim 6$ and 18 for the two sources as averaged over the entire envelope. For these two sources there is a clear anti-correlation between $\mathrm{CO}$ and $\mathrm{CH}_{3} \mathrm{OH}$ gas phase abundances.

To examine whether this anti-correlation is unique to Serpens or a general feature of embedded sources, the outerenvelope $\mathrm{CH}_{3} \mathrm{OH}$ abundances of the sample of Maret et al. (2005) and Jørgensen et al. (2005b) was coupled with CO abundances for the same sources from Jørgensen et al. (2002) (see Fig. 11). The $\mathrm{CO}$ gas phase abundance averaged over the extent of the envelope is taken as a tracer of $\mathrm{CO}$ depletion in the sense that the total gas and ice $\mathrm{CO}$ abundance is assumed to be constant. For sources where the presence of a jump zone can reproduce the observed $\mathrm{CH}_{3} \mathrm{OH}$ emission, only the outer abundance is plotted here. A typical uncertainty of $30 \%$ is assumed both for the $\mathrm{CO}$ and $\mathrm{CH}_{3} \mathrm{OH}$ abundances. Except for three sources (L1157, L1448-I2 and L483) there is a correlation between the two abundances as illustrated by the value of the Pearson correlation coefficient of $r=0.70$. The Serpens sources are characterized by the combination of low CO-abundance and high $\mathrm{CH}_{3} \mathrm{OH}$ abundance. This result implies that the $\mathrm{CH}_{3} \mathrm{OH}$ gas phase abundance is directly related to the current production of $\mathrm{CH}_{3} \mathrm{OH}$ in the outer, cold parts of the envelope, and that any difference from source to source is due to a difference in the amount of $\mathrm{CO}$ frozen out onto the grains. Thus, not all solid $\mathrm{CH}_{3} \mathrm{OH}$ is formed during the cold pre-stellar core phase, consistent with the lack of detected $\mathrm{CH}_{3} \mathrm{OH}$ ice toward background stars behind quiescent dense clouds. This also implies that the lack of $\mathrm{CH}_{3} \mathrm{OH}$ emission at normal cloud positions between the sources is because

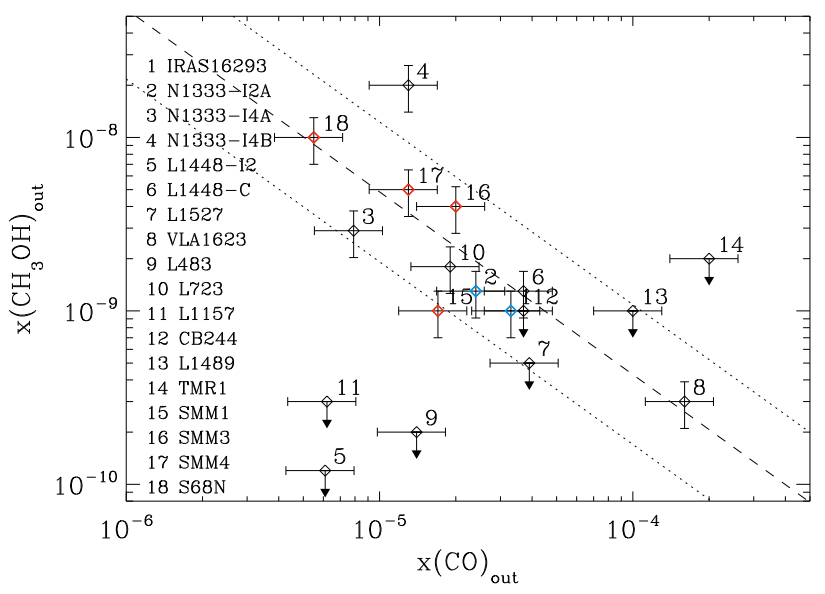

Fig. 11. $\mathrm{CH}_{3} \mathrm{OH}$ gas abundance versus $\mathrm{CO}$ gas abundance for a selection of Class 0 and I sources based on data and analysis from Schöier et al. (2002); Maret et al. (2005); Jørgensen et al. (2005b) and this work. The Serpens sources are in red and IRAS 16293-2422 and NGC 1333IRAS2A are in blue. Sources for which only upper methanol limits exist are marked by arrows. The two dashed lines indicate the $1 \sigma$ confidence limits of the power-law fit.

the density is lower, and hence the timescale for $\mathrm{CO}$ to freeze out is much higher, i.e., the absolute $\mathrm{CH}_{3} \mathrm{OH}$ abundance will drop.

\section{2. $\mathrm{CH}_{3} \mathrm{OH}$ desorption mechanism}

Once methanol has formed on a grain surface it can desorb according to two different mechanisms, thermal and non-thermal desorption. In the first mechanism the entire grain is heated thermally (macroscopic grain heating), and the icy mantle evaporates entirely, releasing all adsorbed species into the gas phase. The ice mantles typically evaporate at grain temperatures of $30-100 \mathrm{~K}$, depending on species. The other mechanism is nonthermal desorption, in which the ice mantle is "heated" on a microscopic (local) scale, either due to absorption of a single UV-photon (Öberg et al. 2009c), impact of a cosmic ray particle (Léger et al. 1985; Hasegawa \& Herbst 1993; Herbst \& Cuppen 2006), or the binding energy being released from the formation of a new molecule (Garrod et al. 2007) or sputtering in outflows (Jiménez-Serra et al. 2008).

\subsubsection{Thermal desorption}

In the case of methanol, the thermal evaporation temperature has been determined experimentally to $80-100 \mathrm{~K}$ (Brown \& Bolina 2007; Green et al. 2009) as is also indicated in Fig. 9. The lower temperature corresponds to evaporation of a pure $\mathrm{CH}_{3} \mathrm{OH}$ ice and the higher to a mix of $\mathrm{CH}_{3} \mathrm{OH}$ and $\mathrm{H}_{2} \mathrm{O}$. Because $\mathrm{CO}$ freezes out on top of a water ice, $\mathrm{CH}_{3} \mathrm{OH}$ is expected to be present in the same layer. Thus the desorption temperature should be close to that of a pure $\mathrm{CH}_{3} \mathrm{OH}$ ice $(\sim 85 \mathrm{~K})$, which is slightly lower, but not much, than that of $\mathrm{CH}_{3} \mathrm{OH}$ mixed with $\mathrm{H}_{2} \mathrm{O}$. This is also found to be the case experimentally (Bisschop 2007). Thus, for the thermal-desorption mechanism to be active, it is necessary that all grains are heated to greater than $80 \mathrm{~K}$, i.e., close to the protostar itself. From the physical structure models above it is predicted that the radius at which $T_{\text {dust }}>80 \mathrm{~K}$ is of the order of 50-100 AU or 0.'2-0.'4 for $d=230 \mathrm{pc}$, depending on source, leading to a beam-dilution factor of 1500-5000. Methanol 
emission originating from very close to the protostar itself has previously been observed for two low-mass sources, IRAS 16293-2422 ( $d=125$ pc; van Dishoeck et al. 1995; Schöier et al. 2002) and NGC 1333 IRAS2A ( $d=250$ pc; Maret et al. 2005). Even though the regions have not been spatially resolved with single-dish data, it has been possible to infer the existence of "hot cores" based on observations of high- $K$ lines $(K>3)$ and measured rotational temperatures of $>80 \mathrm{~K}$. This indicates that the emission arises in a compact, warm and dense region, consistent with it originating close to the protostar itself and also consistent with high-spatial resolution interferometer data (e.g., Jørgensen et al. 2005a, 2007). For the sources presented here, it has only been possible to provide an upper limit on the inner abundance, which in most cases is a few $\times 10^{-7}$ with respect to $\mathrm{H}_{2}$. One notable exception is SMM1, where the upper limit on the inner abundance is close to the outer abundance, a result that will be discussed further below (Sect. 4.3).

\subsubsection{Non-thermal desorption}

Since the temperature in the outer envelope is significantly lower than $100 \mathrm{~K}$, the primary desorption mechanism must be nonthermal. This is supported by the fact that the abundance only follows the column density, and does not appear to depend on temperature. No attempt is made to distinguish between the above mentioned mechanisms here. If desorption is induced by secondary UV photons from cosmic ray ionization of $\mathrm{H}_{2}$ the gas phase abundance is expected to be $10^{-4}-10^{-3}$ times the ice abundance (Öberg et al. 2009a,b). In the case of SMM4 where the $\mathrm{CH}_{3} \mathrm{OH}$-ice abundance is $\sim 3 \times 10^{-5}$ with respect to $\mathrm{H}_{2}$ (Pontoppidan et al. 2004) the UV-induced desorption alone can account for a gas phase abundance of $10^{-9}-10^{-8}$. This is remarkably close to the observed gas phase abundance of $\sim 2 \times 10^{-8}$.

Garrod et al. (2007) modelled the release of $\mathrm{CH}_{3} \mathrm{OH}$ from the grain surface into the gas phase by examining whether the release of binding energy would be enough to evaporate the molecule into the gas phase. They found that the fraction was probably in the range of $1-10 \%$, but could not pin it down any further. In the case of SMM4, results presented here combined with ice observations indicate that much less than $1-10 \%$ is desorbed, since the gas/ice abundance is $\sim 10^{-4}$. Results presented in Hasegawa \& Herbst (1993) show that direct cosmic ray desorption is not an efficient desorption mechanism for tightly bound species like $\mathrm{H}_{2} \mathrm{O}$ and $\mathrm{CH}_{3} \mathrm{OH}$ when compared to the accretion timescale. In fact, they find that the difference between the two rates is of the order of $10^{4}$. Previous studies show that direct cosmic ray induced desorption is most efficient for volatile species (see, e.g., Shen et al. 2004; Roberts et al. 2007) and for species adsorbed on very small grains $(r \leq 0.05 \mu \mathrm{m}$; Herbst \& Cuppen 2006).

\subsubsection{Shock-induced desorption}

In outflows the main desorption mechanism is sputtering of the grain mantle, i.e., impacts of high-temperature gas molecules and atoms, primarily $\mathrm{H}_{2}$ and $\mathrm{He}$. This can in principle be compared to the direct cosmic ray desorption mechanism, except that the flux of $\mathrm{H}_{2}$ and $\mathrm{He}$ is orders of magnitude higher and the impact energy is much lower. The efficiency of this desorption mechanism is clearly seen from the fifth column of Table 5, where the $\mathrm{CH}_{3} \mathrm{OH}$ abundance in the outflow positions is compared to the ambient envelope abundance. In particular, the SMM4-S methanol outflow abundance is $\sim 10^{-5}$ with respect to $\mathrm{H}_{2}$, i.e., only a factor of 3 lower than the measured $\mathrm{CH}_{3} \mathrm{OH}-$ ice abundance in a region located $\sim 15^{\prime \prime}(4000 \mathrm{AU})$ to the north (see Fig. 1 and Pontoppidan et al. 2004). If the envelope iceabundance in this region were also $3 \times 10^{-5}$ prior to the impact of the shock, this would indicate that one third or more of the $\mathrm{CH}_{3} \mathrm{OH}$ ice is sputtered from the grain mantle. However, the measured gas-phase abundance as determined from ${ }^{12} \mathrm{CO}$ emission is an upper limit as briefly discussed above. The true value of the abundance probably lies in the range of $10^{-8}$ to $10^{-6}$ where the lower limit is obtained from $\mathrm{C}^{18} \mathrm{O}$ emission.

\subsection{The nature of the embedded YSOs in Serpens}

Two low-mass YSOs have been confirmed observationally to have the same chemical characteristics as high-mass hot cores, IRAS 16293-2422 and NGC 1333-IRAS2A. These two sources are at the same evolutionary stage as SMM1 based on classical indicators, e.g., $T_{\text {bol }}$. The characteristics are: (1) a large number of detected, saturated, complex organic molecules; and (2) a high rotational temperature for methanol $(>80 \mathrm{~K})$. At the same time, neither of these two envelopes show signs of extended methanol emission (van Dishoeck et al. 1995, Kristensen et al. in prep.). IRAS 16293-2422 is a close binary system surrounded by a circum-binary envelope. The inner parts of this envelope are passively heated to temperatures $>80 \mathrm{~K}$, which, along with small-scale shocks impinging on the inner wall of the envelope (Chandler et al. 2005), give rise to the observed hot-core signature. NGC 1333-IRAS2 is also a binary (possibly triple) system, but the projected separation is greater $\left(\sim 30^{\prime \prime}\right)$. It is believed that the same mechanisms are at play in NGC 1333-IRAS2A causing the hot-core signatures (Maret et al. 2005; Jørgensen et al. 2005b). In the following the Serpens sources, and in particular SMM1, will be compared to these two low-mass hot cores, and differences will be discussed.

There are several possible explanations for the lack of "hot core" characteristics in the Serpens sources which may be categorized in the following manner:

1. Physical: No gas is present close to the source, or no hot gas is present.

2. Chemical: Hot gas is present, but the methanol abundance is very low.

3. Observational: Warm methanol is present close to the source, but either the extent is very small, or emission is optically thick.

Choi (2009) recently reported that SMM1 is a close binary with a projected separation of $\sim 500$ AU. The primary would be the submm source associated with SMM1 (SMM1a), while the binary (SMM1b) would be less embedded. SMM1b can be associated with a source observed by Spitzer at wavelengths shorter than $24 \mu \mathrm{m}$, where SMM1a is not detected. If the projected distance is similar to the actual separation between the two sources, then this could have cleared the inner part of the envelope of gas, explaining the absence of hot gas close to the (sub-mm) source. However, both IRAS 16293-2422 and NGC 1333-IRAS2A have been shown to have inner holes or cavities similar in size to that of SMM1 (Schöier et al. 2002; Jørgensen et al. 2005c), therefore this cannot be the entire explanation.

Recent millimeter interferometry of SMM1 indicates that the region close to the protostar has been cleared of gas. Both Enoch et al. (2009) and van Kempen et al. (2009) report the detection of a resolved disk surrounding SMM1. The disk is unusually massive $\left(\sim 1 M_{\odot}\right)$ and has a modelled radius of up to $300 \mathrm{AU}$ (Enoch et al. 2009), while the inner $500 \mathrm{AU}$ of the envelope have been 
cleared of gas. In such a dense disk, only the upper-most layers will be warm or hot, depending on the distance to the protostar, as the dust extinction grows rapidly towards the midplane of the disk. Thus, the column density of hot methanol will be very low. Through SED modeling of the disk, Enoch et al. (2009) estimate that the inclination is $15^{\circ}$, i.e., nearly face-on. This is in conflict with observations of the $3.6-\mathrm{cm}$ radio jet, which indicate that the outflow is moving very close to the plane of the sky (Rodriguez et al. 1989; Moscadelli et al. 2006). Moreover, the ${ }^{12} \mathrm{CO}$ line profiles are very symmetric along the large-scale outflow, i.e., the molecular outflows are also moving close to the plane of the sky. This would indicate that the disk is seen close to edge-on. If so, then the beam-dilution will be very high implying that any hot part will not be detectable in our single-dish beam. The disk surrounding NGC 1333-IRAS2A is less massive compared to SMM1 ( 0.056 $M_{\odot}$; Jørgensen et al. 2009) and is viewed closer to face-on resulting in less extinction and lower beam dilution. This implies that the high- $K$ emission observed in IRAS 16293-2422 and NGC 1333-IRAS2A is originating in the disk close to the protostar. The actual heating mechanism (passive heating or small-scale shocks) cannot be distinguished with the current observations.

If the inner part of the envelope has not been cleared out of warm gas or if the disk is seen face-on, then it is possible that the abundance of methanol is low. As has been shown by current observations, the $\mathrm{CH}_{3} \mathrm{OH}$ gas-phase abundance is comparatively high in the outer parts of the envelopes, so to decrease the abundance in the inner part of the system, destruction of $\mathrm{CH}_{3} \mathrm{OH}$ must be present. This destruction mechanism must be very efficient if it is to destroy all $\mathrm{CH}_{3} \mathrm{OH}$ in the hot-core parts of the envelope, where the abundance is expected to rise to $>10^{-6}$ w.r.t. $\mathrm{H}_{2}$. $\mathrm{CH}_{3} \mathrm{OH}$ can be destroyed in the gas phase through direct reactions with other species, however the rate coefficients are typically low. An alternative mechanism of both the gas and the ice destruction is UV-dissociation. However, this destruction mechanism must also be at play in IRAS 162932422 and NGC 1333-IRAS2A and there is currently no reason why UV-photodissociation would be more efficient in Serpens sources than in the other two nor that the UV-field is enhanced in SMM1.

Finally, a low-mass hot core may be present in all of the Serpens sources, but not observable. This can be due to beam dilution or due to optical depth effects. The region over which methanol desorbs from the grain surface is expected to be $\sim 100 \mathrm{AU}$ at most, and so beam dilution would be of the order of $10^{3}$. However, the same beam dilution would apply to at least NGC 1333-IRAS2A, which is located at a similar distance of $250 \mathrm{pc}$, and can thus not be used as an argument. The line optical depth has been calculated in the RATRAN simulations, and is typically of the order of 0.1 or less for the transitions observed here, even for lines arising in the inner-most part of the envelope. The dust opacity at $338 \mathrm{GHz}$ is less than 0.08 at all times, as estimated from the DUSTY modelling.

Of the three explanations presented above the first is the more plausible if the distance is indeed $230 \mathrm{pc}$ as assumed. Recent VLBA observations indicate that the distance may be closer to 415 pc (Dzib et al. 2010), in which case the third explanation is more plausible. The other two reasons can be disproved through comparison with IRAS 16293-2422 and NGC 1333IRAS2A. However, only SMM1 has been suggested to have a massive disk, and it is also the source that resembles the two lowmass hot cores the most in terms of luminosity. The other sources (SMM3, SMM4 and S68N) have considerably lower luminosities by factors $4-10$. Thus it may be that the hot-core regions around these sources are indeed much smaller and beam-diluted $\left(\sim 5 \times 10^{3}\right)$, comparable to several of the low-mass sources in the sample of Maret et al. (2005) and Jørgensen et al. (2005b). With the current observations only upper limits have been determined of the molecular abundance in the inner envelope of the lower-luminosity sources.

\section{Summary}

Maps of rotationally excited methanol in the Serpens Molecular Core have been presented. Emission arises from the molecular envelopes of four deeply embedded sources and their associated outflows. In particular, three outflow knots have been identified based on their strong methanol emission. Mapping shows methanol emission in all of the four envelopes to be extended out to ranges of $\sim 10000 \mathrm{AU}$. The abundance is constant in the outer parts of the envelope with a value of $10^{-9}-10^{-8}$, depending on source. The methanol abundance at outflow positions is enhanced by up to 2-3 orders of magnitude with respect to the ambient abundance. The measured envelope abundances are consistent with non-thermal desorption of solid-state methanol, through, for example, UV-photodesorption. At outflow positions the enhanced abundance may be explained by sputtering of the grain mantles. The symmetric, slightly shifted line profiles point to the outflows being either caused by J-type shocks or moving along the plane of the sky.

The CO gas abundance is found to be anti-correlated with the methanol gas abundance in the Serpens maps. This result has been extended to literature data of a large sample of other, similarly deeply embedded sources. The reason is that the more $\mathrm{CO}$ is frozen out from the gas phase, the higher the $\mathrm{CO}$ ice abundance, and this adsorbed $\mathrm{CO}$ is then converted to $\mathrm{CH}_{3} \mathrm{OH}$ ice on the grain surface from where it desorbs non-thermally. The non-thermal desorption mechanism implies that the gas-phase abundance follows the solid-state abundance closely, something which has directly shown to be the case here. Thus, the differences in abundance between the four sources can be directly related to the current production of $\mathrm{CH}_{3} \mathrm{OH}$ and reflects how much $\mathrm{CO}$ is frozen out now rather than in some possible colder past.

The Serpens sources do not contain the chemical signatures of low-mass hot cores such as IRAS 16293-2422 and NGC 1333IRAS2A. In the case of SMM1, the more luminous of the Serpens sources and resembling most closely the two low-mass hot cores, the most likely explanation is that it harbours a massive disk which is seen close to edge-on. The other three Serpens sources are all lower in mass and luminosity, and here the absence of a hot-core signature could be ascribed to beam dilution, as also holds for the majority of the deeply embedded sources.

In conclusion, the $\mathrm{CH}_{3} \mathrm{OH}$ emission is found to trace the following: (1) energetic input into cold gas, primarily through outflow interaction; (2) column density of cold gas in the outer envelope; (3) reactions and subsequent desorption of grain surface products.

Because water closely resembles methanol in the sense that it is exclusively formed on grains and it desorbs from grain surfaces at $T>100 \mathrm{~K}$, it is expected that the abundance structure will resemble that of methanol. This is to be tested with upcoming water-observations of these sources as part of the Herschel Guaranteed Time Key Project "Water in Star-forming Regions with Herschel" (WISH).

Acknowledgements. Astrochemistry at Leiden Observatory is supported by a Spinoza prize and by NWO grant 614.041.004. The authors would like to thank the staff at the JCMT for technical help. Floris van der Tak is thanked for help with updating the LAMDA database and Karin Öberg for very stimulating disucssions. T.v.K. is grateful to the SMA for supporting his research at the CfA. 
L. E. Kristensen et al.: Methanol maps of low-mass protostellar systems. I.

Appendix A: SED data points

Table A.1. SED points used for DUSTY modelling.

\begin{tabular}{|c|c|c|c|c|c|c|c|c|c|c|c|c|c|c|c|}
\hline \multicolumn{4}{|c|}{ SMM1 } & \multicolumn{4}{|c|}{ SMM3 } & \multicolumn{4}{|c|}{ SMM4 } & \multicolumn{4}{|c|}{ S68N } \\
\hline $\begin{array}{r}\lambda \\
(\mu \mathrm{m})\end{array}$ & $\begin{array}{r}F \\
(\mathrm{Jy})\end{array}$ & $\begin{array}{r}\text { Beam } \\
\left({ }^{\prime \prime}\right)\end{array}$ & Ref. & $\begin{array}{r}\lambda \\
(\mu \mathrm{m})\end{array}$ & $\begin{array}{r}F \\
(\mathrm{Jy})\end{array}$ & $\begin{array}{r}\text { Beam } \\
\left({ }^{\prime \prime}\right)\end{array}$ & Ref. & $\begin{array}{r}\lambda \\
(\mu \mathrm{m})\end{array}$ & $\begin{array}{r}F \\
(\mathrm{Jy})\end{array}$ & $\begin{array}{r}\text { Beam } \\
\left({ }^{\prime \prime}\right)\end{array}$ & Ref. & $\begin{array}{r}\lambda \\
(\mu \mathrm{m})\end{array}$ & $\begin{array}{r}F \\
(\mathrm{Jy})\end{array}$ & $\begin{array}{r}\text { Beam } \\
\left({ }^{\prime \prime}\right)\end{array}$ & Ref. \\
\hline 70.1 & 313 & 90 & 1 & 24 & 0.109 & & 6 & 60 & 12 & 60 & 2 & 50 & 16 & 32 & 8 \\
\hline 73.6 & 353 & 90 & 1 & 60 & 22 & 60 & 2 & 100 & 60 & 60 & 2 & 60 & 8 & 60 & 2 \\
\hline 77.3 & 402 & 90 & 1 & 70 & 12.2 & & 6 & 450 & 10.8 & 8 & 3 & 70 & 14.9 & & 6 \\
\hline 81.2 & 445 & 90 & 1 & 100 & 70 & 100 & 2 & 800 & 4.13 & 14 & 4 & 70 & 5.664 & & 9 \\
\hline 85.2 & 489 & 90 & 1 & 450 & 7.1 & 8 & 3 & 850 & 2.9 & 14 & 3 & 100 & 37 & 100 & 2 \\
\hline 89.5 & 536 & 90 & 1 & 800 & 2.01 & 14 & 4 & 1100 & 1.47 & 19 & 4 & 450 & 25 & 8 & 10 \\
\hline 94.0 & 573 & 90 & 1 & 850 & 1.5 & 14 & 3 & 1300 & 0.92 & 22 & 4 & 450 & 9.1 & 8 & 3 \\
\hline 98.7 & 612 & 90 & 1 & 1100 & 1.11 & 19 & 4 & 1400 & 1.108 & 1 & 7 & 850 & 1.6 & 14 & 3 \\
\hline 103.6 & 638 & 90 & 1 & 1300 & 0.92 & 22 & 4 & 2000 & 0.6 & 35 & 4 & 850 & 3.3 & 20 & 10 \\
\hline 108.8 & 661 & 90 & 1 & 1400 & 0.651 & 2 & 7 & 2700 & 0.143 & 2 & 7 & 1100 & 2.32 & 40 & 9 \\
\hline 114.2 & 685 & 90 & 1 & 2700 & 0.09 & 4 & 7 & 3200 & 0.097 & 4 & 7 & 1100 & 3.78 & 40 & 9 \\
\hline 119.9 & 698 & 90 & 1 & 3400 & 0.043 & 5 & 7 & 3400 & 0.075 & 5 & 7 & 1250 & 0.9 & 32 & 8 \\
\hline 125.9 & 713 & 90 & 1 & & & & & & & & & 2727 & 0.0361 & 7 & 11 \\
\hline 132.2 & 733 & 90 & 1 & & & & & & & & & 3100 & 0.036 & 32 & 8 \\
\hline 138.8 & 760 & 90 & 1 & & & & & & & & & & & & \\
\hline 145.8 & 770 & 90 & 1 & & & & & & & & & & & & \\
\hline 153.1 & 785 & 90 & 1 & & & & & & & & & & & & \\
\hline 160.7 & 785 & 90 & 1 & & & & & & & & & & & & \\
\hline 100 & 435 & 100 & 2 & & & & & & & & & & & & \\
\hline 450 & 35.7 & 8 & 3 & & & & & & & & & & & & \\
\hline 850 & 6.1 & 14 & 3 & & & & & & & & & & & & \\
\hline 1100 & 3.47 & 19 & 4 & & & & & & & & & & & & \\
\hline 1300 & 2.52 & 22 & 4 & & & & & & & & & & & & \\
\hline 2000 & 0.91 & 35 & 4 & & & & & & & & & & & & \\
\hline 3000 & 0.233 & 5 & 5 & & & & & & & & & & & & \\
\hline
\end{tabular}

References. (1) Larsson et al. (2000), Larsson, priv. comm. (2) Hurt \& Barsony (1996) (3) Davis et al. (1999); (4) Casali et al. (1993); (5) Testi \& Sargent (1998); (6) Harvey et al. (2007); (7) Hogerheijde et al. (1999); (8) McMullin et al. (1994); (9) Enoch et al. (2007); (10) Wolf-Chase et al. (1998); (11) Williams \& Myers (2000).

\section{References}

Bachiller, R., \& Pérez Gutiérrez, M. 1997, ApJ, 487, 93

Bachiller, R., \& Tafalla, M. 1999, in The Origin of Stars and Planetary Systems, ed. C. J. Lada, \& N. D. Kylafis, NATO ASIC Proc. 540, 227

Bachiller, R., Liechti, S., Walmsley, C. M., \& Colomer, F. 1995, A\&A, 295, 51

Bachiller, R., Codella, C., Colomer, F., Liechti, S., \& Walmsley, C. M. 1998, A\&A, 335, 266

Bergin, E. A., Alves, J., Huard, T., \& Lada, C. J. 2002, ApJ, 570, L101

Bisschop, S. E. 2007, Ph.D. Thesis, Leiden, The Netherlands

Blake, G. A., Sandell, G., van Dishoeck, E. F., et al. 1995, ApJ, 441, 689

Brown, W. A., \& Bolina, A. S. 2007, MNRAS, 374, 1006

Casali, M. M., Eiroa, C., \& Duncan, W. D. 1993, A\&A, 275, 195

Ceccarelli, C., Loinard, L., Castets, A., Tielens, A. G. G. M., \& Caux, E. 2000, A\&A, 357, L9

Chandler, C. J., Brogan, C. L., Shirley, Y. L., \& Loinard, L. 2005, ApJ, 632, 371

Choi, M. 2009, ApJ, 705, 1730

Cuppen, H. M., van Dishoeck, E. F., Herbst, E., \& Tielens, A. G. G. M. 2009, A\&A, 508, 275

Dartois, E., Schutte, W., Geballe, T. R., et al. 1999, A\&A, 342, L32

Davis, C. J., Matthews, H. E., Ray, T. P., Dent, W. R. F., \& Richer, J. S. 1999 , MNRAS, 309, 141

Di Francesco, J., Johnstone, D., Kirk, H., MacKenzie, T., \& Ledwosinska, E. 2008, ApJS, 175, 277

Dzib, S., Loinard, L., Mioduszewski, A. J., et al. 2010, ApJ, accepted [arXiv: 1003.5900$]$

Eiroa, C., Djupvik, A. A., \& Casali, M. M. 2008, in The Southern Sky ASP Monograph Publications, ed. B. Reipurth, Handbook of Star Forming Regions, II, 5, 693

Enoch, M. L., Glenn, J., Evans, II, N. J., et al. 2007, ApJ, 666, 982

Enoch, M. L., Corder, S., Dunham, M. M., \& Duchêne, G. 2009, ApJ, 707, 103

Evans, N. J., Dunham, M. M., Jørgensen, J. K., et al. 2009, ApJS, 181, 321

Friberg, P., Hjalmarson, A., Madden, S. C., \& Irvine, W. M. 1988, A\&A, 195 281

Froebrich, D. 2005, ApJS, 156, 169
Fuchs, G. W., Cuppen, H. M., Ioppolo, S., et al. 2009, A\&A, 505, 629

Garay, G., Mardones, D., \& Rodríguez, L. F. 2000, ApJ, 545, 861

Garay, G., Mardones, D., Rodríguez, L. F., Caselli, P., \& Bourke, T. L. 2002, ApJ, 567, 980

Garrod, R., Park, I. H., Caselli, P., \& Herbst, E. 2006, in Faraday Discussions 133,51

Garrod, R. T., Wakelam, V., \& Herbst, E. 2007, A\&A, 467, 1103

Gibb, E. L., Whittet, D. C. B., Boogert, A. C. A., \& Tielens, A. G. G. M. 2004, ApJS, 151, 35

Graves, S. F., Richer, J. S., Buckle, J. V., et al. 2010, MNRAS, accepted [arXiv: 1006.0891$]$

Green, S. D., Bolina, A. S., Chen, R., et al. 2009, MNRAS, 398, 357

Harvey, P., Merín, B., Huard, T. L., et al. 2007, ApJ, 663, 1149

Hasegawa, T. I., \& Herbst, E. 1993, MNRAS, 261, 83

Herbst, E., \& Cuppen, H. M. 2006, Proceedings of the National Academy of Science, 103, 12257

Hiraoka, K., Sato, T., Sato, S., et al. 2002, ApJ, 577, 265

Hogerheijde, M. R., \& van der Tak, F. F. S. 2000, A\&A, 362, 697

Hogerheijde, M. R., van Dishoeck, E. F., Salverda, J. M., \& Blake, G. A. 1999, ApJ, 513, 350

Hollenbach, D. 1997, in Herbig-Haro Flows and the Birth of Stars, ed. B. Reipurth, \& C. Bertout, IAU Symp., 182, 181

Hurt, R. L., \& Barsony, M. 1996, ApJ, 460, L45

Ivezic, Z., \& Elitzur, M. 1997, MNRAS, 287, 799

Jiménez-Serra, I., Caselli, P., Martín-Pintado, J., \& Hartquist, T. W. 2008, A\&A, 482,549

Jørgensen, J. K., Schöier, F. L., \& van Dishoeck, E. F. 2002, A\&A, 389, 908 Jørgensen, J. K., Schöier, F. L., \& van Dishoeck, E. F. 2004, A\&A, 416, 603 Jørgensen, J. K., Bourke, T. L., Myers, P. C., et al. 2005a, ApJ, 632, 973 Jørgensen, J. K., Schöier, F. L., \& van Dishoeck, E. F. 2005b, A\&A, 437, 501 Jørgensen, J. K., Schöier, F. L., \& van Dishoeck, E. F. 2005c, A\&A, 435, 177 Jørgensen, J. K., Bourke, T. L., Myers, P. C., et al. 2007, ApJ, 659, 479 Jørgensen, J. K., van Dishoeck, E. F., Visser, R., et al. 2009, A\&A, 507, 861 Larsson, B., Liseau, R., Men'shchikov, A. B., et al. 2000, A\&A, 363, 253

Léger, A., Jura, M., \& Omont, A. 1985, A\&A, 144, 147 
Leurini, S., Schilke, P., Wyrowski, F., \& Menten, K. M. 2007, A\&A, 466, 215 Maret, S., Ceccarelli, C., Tielens, A. G. G. M., et al. 2005, A\&A, 442, 527 McMullin, J. P., Mundy, L. G., Blake, G. A., et al. 2000, ApJ, 536, 845

McMullin, J. P., Mundy, L. G., Wilking, B. A., Hezel, T., \& Blake, G. A. 1994, ApJ, 424, 222

Moscadelli, L., Testi, L., Furuya, R. S., et al. 2006, A\&A, 446, 985

Müller, H. S. P., Thorwirth, S., Roth, D. A., \& Winnewisser, G. 2001, A\&A, 370, L49

Öberg, K. I., Bottinelli, S., \& van Dishoeck, E. F. 2009a, A\&A, 494, L13

Öberg, K. I., Garrod, R. T., van Dishoeck, E. F., \& Linnartz, H. 2009b, A\&A, 504,891

Öberg, K. I., van Dishoeck, E. F., \& Linnartz, H. 2009c, A\&A, 496, 281

Ossenkopf, V., \& Henning, T. 1994, A\&A, 291, 943

Pontoppidan, K. M. 2006, A\&A, 453, L47

Pontoppidan, K. M., van Dishoeck, E. F., \& Dartois, E. 2004, A\&A, 426, 925

Pontoppidan, K. M., Boogert, A. C. A., Fraser, H. J., et al. 2008, ApJ, 678, 1005

Pottage, J. T., Flower, D. R., \& Davis, S. L. 2004, MNRAS, 352, 39

Roberts, J. F., Rawlings, J. M. C., Viti, S., \& Williams, D. A. 2007, MNRAS, 382,733

Rodriguez, L. F., Curiel, S., Moran, J. M., et al. 1989, ApJ, 346, L85

Schnee, S. L., Ridge, N. A., Goodman, A. A., \& Li, J. G. 2005, ApJ, 634, 442
Schöier, F. L., Jørgensen, J. K., van Dishoeck, E. F., \& Blake, G. A. 2002, A\&A, 390, 1001

Schöier, F. L., van der Tak, F. F. S., van Dishoeck, E. F., \& Black, J. H. 2005, A\&A, 432, 369

Shen, C. J., Greenberg, J. M., Schutte, W. A., \& van Dishoeck, E. F. 2004, A\&A, 415, 203

Smith, H., Hills, R. E., Withington, S., et al. 2003, in Society of PhotoOptical Instrumentation Engineers (SPIE) Conference, ed. T. G. Phillips, \& J. Zmuidzinas, 4855, 338

Tafalla, M., Myers, P. C., Mardones, D., \& Bachiller, R. 2000, A\&A, 359, 967 Testi, L., \& Sargent, A. I. 1998, ApJ, 508, L91

van der Tak, F. F. S., van Dishoeck, E. F., \& Caselli, P. 2000, A\&A, 361, 327

van Dishoeck, E. F., \& Blake, G. A. 1998, ARA\&A, 36, 317

van Dishoeck, E. F., Blake, G. A., Jansen, D. J., \& Groesbeck, T. D. 1995, ApJ, 447, 760

van Kempen, T. A., Wilner, D., \& Gurwell, M. 2009, ApJ, 706, L22

Watanabe, N., \& Kouchi, A. 2002, ApJ, 571, L173

White, G. J., Casali, M. M., \& Eiroa, C. 1995, A\&A, 298, 594

Williams, J. P., \& Myers, P. C. 2000, ApJ, 537, 891

Wilson, T. L., \& Rood, R. 1994, ARA\&A, 32, 191

Wolf-Chase, G. A., Barsony, M., Wootten, H. A., et al. 1998, ApJ, 501, L193 